\title{
LOCAL SOLUTIONS FOR STOCHASTIC NAVIER STOKES EQUATIONS*
}

\author{
Alain Bensoussan $^{1}$ And Jens Frehse ${ }^{2}$
}

\begin{abstract}
In this article we consider local solutions for stochastic Navier Stokes equations, based on the approach of Von Wahl, for the deterministic case. We present several approaches of the concept, depending on the smoothness available. When smoothness is available, we can in someway reduce the stochastic equation to a deterministic one with a random parameter. In the general case, we mimic the concept of local solution for stochastic differential equations.
\end{abstract}

Mathematics Subject Classification. 35Q30, 76D06.

Received: December 2, 1999.

\section{INTRODUCTION}

The usual approach to stochastic Navier Stokes equations is related to proving the existence of strong or weak global solutions (namely in any compact interval of time), see references $[1,2,4]$, and related papers. Strong solutions are obtained in very limited situations. In general, one relies on a background deterministic theory which is the variational theory, see references $[7,8]$. As in the deterministic case, one considers a Galerkin approximation method, and by a fundamental property of the nonlinear term, it drops out in writing the energy equality. Therefore estimates can be obtained. Compactness is then necessary to proceed. In the stochastic case, the compactness holds only in spaces of probability measures related to the trajectories and not in functional spaces related to the trajectories themselves as in the deterministic case. Nevertheless, this is sufficient to prove the existence of weak solutions. An other approach to Navier Stokes equations is that of Von Wahl [9]. It relies on abstract parabolic equations and obtains more regular solutions, but only locally (on a small interval of time). Such solutions are also unique. The objective of this paper is to use such a deterministic background to construct solutions to stochastic Navier Stokes equations locally. In cases, which to some extent are reducible to the deterministic case (namely stochastic integrals of Ito type can be removed) then we can proceed in a way similar to that of Von Wahl. A slight improvement is however used in comparison to the deterministic theory, where solutions which are differentiable in time are possible. Because of the stochastic context, only solutions which are hölderian in time are possible here.

To treat equations of Ito type, we approach differently the concept of local solutions, in a way which is usual in the stochastic context, for ordinary stochastic differential equations (see [5]). The method can also be used to treat local solutions for deterministic equations, but it is not needed in the deterministic context. We show

\footnotetext{
Keywords and phrases. Navier Stokes equations, stochastic equations, abstract parabolic equations, Ito integral, local solution, Ito equation, Stokes operator, Functional equation, Mild solution, Random time.

* Dedicated to Roger Temam for his 60th birthday

1 University Paris Dauphine and CNES, 2 Place Maurice Quantor, 75001 Paris, France.

2 Institüt für Angewandte Mathematik, Universität Bonn, 6 BeringStrasse, Bonn, Germany.
} 
that it fits perfectly to Navier Stokes equations of Ito type, and provides a result of existence and uniqueness of a strong local solution.

\section{Functional BACKGROUnd}

\subsection{Notation}

Let $\mathcal{O}$ be an open domain of $\mathbb{R}^{n}$ with regular boundary $\partial \mathcal{O}$. Let $\mathcal{V}$ be the space of infinitely differentiable $n$-dimensional vector fields $u(x)$ on $\mathcal{O}$ with compact support strictly contained in $\mathcal{O}$, satisfying $\operatorname{div} u(x)=0$. We call $H$ the closure of $\mathcal{V}$ in $\left(L^{2}(\mathcal{O})\right)^{n}$, and denote by $P$ the projection from $\left(L^{2}(\mathcal{O})\right)^{n}$ into $H$. We also call

$$
V=\left\{u \text { in }\left(H_{0}^{1}(\mathcal{O})\right)^{n}, \operatorname{div} u=0\right\} .
$$

We consider the linear operator

$$
A u=-P \Delta u
$$

with

$$
D(A)=\left(H^{2}(\mathcal{O})\right)^{n} \cap V .
$$

Note that $A$ is a positive self adjoint operator in $H$ and one has

$$
(A u, u)=\|u\|_{V}^{2} .
$$

Considering the fractional powers $A^{\gamma}$, with $0 \leq \gamma \leq 1$, one has the properties

$$
\begin{gathered}
D\left(A^{\frac{1}{2}}\right)=V \\
D\left(A^{\gamma}\right) \subset\left(H^{2 \gamma}(\mathcal{O})\right)^{n} \cap H, \quad 0 \leq \gamma \leq 1 .
\end{gathered}
$$

with continuous injection. Moreover

$$
D\left(A^{\gamma}\right)=\left(H_{0}^{2 \gamma}(\mathcal{O})\right)^{n} \cap H, \quad 0 \leq \gamma \leq \frac{1}{2}, \gamma \neq \frac{1}{4}
$$

and

$$
\left(H_{0}^{2 s}(\mathcal{O})\right)^{n} \cap H \subset D\left(A^{\gamma}\right), \frac{1}{2}<\gamma<s \leq 1, s \neq \frac{3}{4} .
$$

Also

$$
\left(H^{2 \gamma}(\mathcal{O})\right)^{n} \cap H=D\left(A^{\gamma}\right), 0 \leq \gamma<\frac{1}{4} .
$$

For the proofs, we refer to [9]. The operator $A$ is called the Stokes operator, and $-A$ generates an analytic semi group, denoted by $e^{-A t}$. More precisely one has the properties

$$
\left\|(\lambda+A)^{-1}\right\| \leq \frac{M}{|\lambda|+1}, \quad \text { Re } \lambda \geq 0
$$




$$
\left\|A^{1-\rho} e^{-A t}\right\| \leq \frac{c(\delta, M) e^{-\delta t}}{t^{1-\rho}}, \quad 0 \leq \rho \leq 1, \delta<\frac{1}{M}
$$

Note that if $f \in H$ and

$$
A u=f
$$

then there exists an element $\pi \in H^{1}(\mathcal{O})$ uniquely defined up to a constant such that

$$
-\Delta u+D \pi=f
$$

The function $\pi$ is called the pressure.

\subsection{Auxiliary results}

We first recall a useful result. We consider the integral

$$
\psi(t)=\int_{0}^{t} e^{-A(t-s)} g(s) \mathrm{d} s .
$$

We note $C^{\beta}([0, T] ; H)$ the space of functions of time with value in $H$, which are Hölder with exponent $\beta$, with the norm

$$
\|g\|_{C^{\beta}([0, T] ; H)}=\sup _{\{t \in[0, T]\}}|g(t)|+\sup _{\left\{t, t^{\prime} \in[0, T]\right\}} \frac{\left|g(t)-g\left(t^{\prime}\right)\right|}{\left|t-t^{\prime}\right|^{\beta}}
$$

then we have the

Proposition 2.1. If $g \in C^{\beta}([0, T] ; H)$, then one has

$$
\psi \in C^{1}([0, T] ; H) \cap C^{0}([0, T] ; D(A))
$$

with the estimate

$$
\|\psi\|_{C^{1}([0, T] ; H) \cap C^{0}([0, T] ; D(A))} \leq c\left(\|g\|_{C^{0}([0, T] ; H)}+\|g\|_{C^{\beta}([0, T] ; H)} \frac{T^{\beta}}{\beta}\right)
$$

where the constant $c$ does not depend on $\beta$.

Proof.

Note first the formula

$$
A \psi(t)=\int_{0}^{t} A e^{-A(t-s)}(g(s)-g(t)) \mathrm{d} s+g(t)-e^{-A t} g(t) .
$$

The function $g(t)-e^{-A t} g(t)$ is clearly in $C^{0}([0, T] ; H)$. Moreover

$$
\left|\int_{0}^{t} A e^{-A(t-s)}(g(s)-g(t)) \mathrm{d} s\right| \leq c \frac{\|g\|_{C^{\beta}([0, T] ; H)}}{\beta} .
$$

Thanks to this estimate it is thus sufficient to prove the continuity in time of the function

$$
\int_{0}^{t} A e^{-A(t-s)}(g(s)-g(t)) \mathrm{d} s .
$$


But for $0 \leq t \leq T-h$, one has

$$
\begin{aligned}
& \int_{0}^{t+h} A e^{-A(t+h-s)}(g(s)-g(t+h)) \mathrm{d} s-\int_{0}^{t} A e^{-A(t-s)}(g(s)-g(t)) \mathrm{d} s=\int_{t}^{t+h} A e^{-A(t+h-s)}(g(s)-g(t+h)) \mathrm{d} s \\
& +\int_{0}^{t}\left(A e^{-A(t+h-s)}(g(s)-g(t+h))-A e^{-A(t-s)}(g(s)-g(t))\right) \mathrm{d} s .
\end{aligned}
$$

The first integral is estimated by

$$
c \frac{h^{\beta}}{\beta}\|g\|_{C^{\beta}([0, T] ; H)} .
$$

In the second we write the integrand as

$$
\left(e^{-A h}-I\right) A e^{-A(t-s)}(g(s)-g(t))-A e^{-A(t+h-s)}(g(t+h)-g(t))
$$

and for $s<t$, it is easy to see that it converges to 0 in $H$ as $h \rightarrow 0$. Moreover, thanks to the Hölder continuity of $g$, we can bound the norm of the integrand by a function of $s$ not depending on $h$, which is integrable. One may then apply Lebesgue's Theorem to conclude that $A \psi(t)$ belongs to $C^{0}([0, T] ; H)$ and

$$
\|\psi\|_{C^{0}([0, T] ; D(A))} \leq c\left(\|g\|_{C^{0}([0, T] ; H)}+\frac{\|g\|_{C^{\beta}([0, T] ; H)}}{\beta}\right) .
$$

Next one writes

$$
\frac{\psi(t+h)-\psi(t)}{h}=\int_{0}^{1} e^{-h \tau A} \mathrm{~d} \tau g(t)+\frac{\left(e^{-A h}-I\right)}{h} \psi(t)+\frac{1}{h} \int_{t}^{t+h} e^{-A(t+h-s)}(g(s)-g(t)) \mathrm{d} s .
$$

Since $\psi(t)$ belongs to $D(A)$, we can pass to the limit in the right hand side, as $h \rightarrow 0$, to obtain

$$
\psi^{\prime}(t)=g(t)-A \psi(t) .
$$

Thanks to the previous estimate, we can conclude the proof of $(2.8)$.

Corollary 2.1. We have also

$$
\psi \in C^{\rho}\left([0, T] ; D\left(A^{1-\rho}\right)\right)
$$

with the same estimate as (2.8).

Proof.

One can write for $0 \leq t \leq T-h$

$$
A^{1-\rho}(\psi(t+h)-\psi(t))=\int_{t}^{t+h} A^{1-\rho} e^{-A(t+h-s)} g(s) \mathrm{d} s+A^{1-\rho}\left(e^{-h A}-I\right) \psi(t) .
$$

The norm of the integral in the right hand side is estimated by $h^{\rho}\|g\|_{C^{0}([0, T] ; H)}$. The norm of the second term is estimated by $h^{\rho}\|A \psi\|_{C^{0}([0, T] ; H)}$ and the result follows.

Note also the result, whose proof is obtained by similar methods. 
Proposition 2.2. If $g \in C^{0}([0, T] ; H)$, then one has

$$
\psi \in C^{\delta}\left([0, T] ; D\left(A^{1-\rho}\right)\right), \forall \delta<\rho
$$

with the estimate, valid for $0<\delta<\rho$

$$
\|\psi\|_{C^{\delta}\left([0, T] ; D\left(A^{1-\rho}\right)\right)} \leq c\|g\|_{C^{0}([0, T] ; H)} T^{\rho-\delta}\left(\frac{1}{\rho}+\frac{1}{\delta(\rho-\delta)}\right)
$$

where the constant $c$ does not depend on $\rho$, or on $\delta$. For $\delta=0$, one should use the estimate

$$
\|\psi\|_{C^{0}\left([0, T] ; D\left(A^{1-\rho}\right)\right)} \leq c\|g\|_{C^{0}([0, T] ; H)} \frac{T^{\rho}}{\rho} .
$$

We then proceed with properties of the vorticity operator. The vorticity operator is defined by the formula

$$
B(u)=P(u \cdot D u)
$$

which makes sense at least for vector fields $u$ such that $u$. $D u \in\left(L^{2}(\mathcal{O})\right)^{n}$. We want to prove the following important result

Proposition 2.3. Assume $n<6$. Let

$$
0<\rho<\min \left(\frac{1}{2}, \frac{3}{4}-\frac{n}{8}\right)
$$

then $B(u)$ maps $D\left(A^{1-\rho}\right)$ into $H$ and one has the estimate

$$
|B(u)-B(v)| \leq c\left(\left|A^{1-\rho} u\right|+\left|A^{1-\rho} v\right|\right)\left|A^{1-\rho}(u-v)\right|
$$

Proof.

Set $\gamma=1-\rho$, then

$$
1>\gamma>\max \left(\frac{1}{2}, \frac{1}{4}+\frac{n}{8}\right)
$$

We first check that if

$$
\phi, \psi \in\left(H^{2 \gamma}(\mathcal{O})\right)^{n}
$$

then

$$
\phi . D \psi \in\left(L^{2}(\mathcal{O})\right)^{n}
$$

and

$$
|\phi . D \psi|_{\left(L^{2}(\mathcal{O})\right)^{n}} \leq c|\phi|_{\left(H^{2 \gamma}(\mathcal{O})\right)^{n}}|\psi|_{\left(H^{2 \gamma}(\mathcal{O})\right)^{n}} .
$$

Indeed, we can find $q_{1}$ such that

$$
\left(1-\frac{4}{n}\right)^{+}<\frac{1}{q_{1}}<\min \left(1, \frac{4 \gamma-2}{n}\right) .
$$


Therefore

$$
\frac{1}{2 q_{1}}>\frac{1}{2}-\frac{2 \gamma}{n}
$$

and

$$
\frac{1}{2 q_{2}}=\frac{1}{2}-\frac{1}{2 q_{1}}>\frac{1}{2}-\frac{2 \gamma-1}{n}
$$

By Sobolev embedding, we then have

$$
\begin{aligned}
\left.H^{2 \gamma} \mathcal{O}\right) & \subset W^{1,2 q_{2}}(\mathcal{O}) \\
H^{2 \gamma}(\mathcal{O}) & \subset L^{2 q_{1}}(\mathcal{O})
\end{aligned}
$$

but

$$
|\phi . D \psi|_{\left(L^{2}(\mathcal{O})\right)^{n}} \leq c|\phi|_{\left(L^{\left.2 q_{1}(\mathcal{O})\right)^{n}}\right.}|\psi|_{\left(W^{\left.1,2 q_{2}(\mathcal{O})\right)^{n}}\right.}
$$

which implies (2.14).

Now we can assert that if

$$
\phi, \psi \in\left(H^{2 \gamma}(\mathcal{O})\right)^{n} \cap H
$$

then

$$
|P(\phi . D \psi)| \leq|\phi . D \psi|_{\left(L^{2}(\mathcal{O})\right)^{n}} \leq c|\phi|_{\left(H^{2 \gamma}(\mathcal{O})\right)^{n} \cap H}|\psi|_{\left(H^{2 \gamma}(\mathcal{O})\right)^{n} \cap H}
$$

and from (2.1) it follows

$$
|P(\phi . D \psi)| \leq c\left|A^{\gamma} \phi\right|\left|A^{\gamma} \psi\right|
$$

Then (2.14) follows easily.

Remark 2.1. In [9] it is proven that

$$
|B(u)-B(v)| \leq c(|A u|+|A v|)\left|A^{1-\rho}(u-v)\right|
$$

which is a less good estimate than (2.14). The improvement is minor in the deterministic context, although it simplifies the proofs of [9]. More importantly, it is very useful for the type of equations we are going to consider in the sequel.

\section{LOCAL SOLUTION OF AN ABSTRACT PARABOLIC EQUATION}

\subsection{Setting of the problem and statement of results}

We consider a function $\phi(t)$ with values in $D\left(A^{1-\rho}\right)$ such that

$$
\phi \in C^{\beta}\left(\left[0, T_{0}\right] ; D\left(A^{1-\rho}\right)\right)
$$


Note that the function

$$
\sigma(t)=\frac{t^{\rho}}{\rho} \sup _{0 \leq s \leq t}\left|A^{1-\rho} \phi(s)\right|
$$

is continuous in $t$ on $\left[0, T_{0}\right]$ and increasing. Let $T$ be defined by

$$
\begin{aligned}
& T=T_{0}, \text { if } c \sigma\left(T_{0}\right) \leq 1 \\
& T=\min \left\{0<t<T_{0} \mid c \sigma(t) \geq 1\right\}, \text { if } c \sigma\left(T_{0}\right)>1
\end{aligned}
$$

where $c$ is some constant related only to those appearing in (2.14) and in (2.6). We shall designate by the same notation $c$ such constants. We then state the following

Theorem 3.1. Assume (3.1), then there exists a unique $z$ such that

$$
z \in C^{1}([0, T] ; H) \cap C^{0}([0, T] ; D(A)) z^{\prime}+A z+B(z+\phi)=0, \forall t \in[0, T] z(0)=0 .
$$

Remark 3.1. If $\operatorname{co}\left(T_{0}\right) \leq 1$, then we can take $T=T_{0}$. So we can solve (3.3) globally on $\left[0, T_{0}\right]$, provided the data is sufficiently small.

Remark 3.2. In fact, if we cannot solve $(3.1)$ on $\left[0, T_{0}\right]$, it means that there exists $\hat{T} \leq T_{0}$, which will be the explosion time of $\left|A^{1-\rho} z(t)\right|$ on $\left[0, T_{0}\right]$, namely

$$
\lim t \uparrow \hat{T}\left|A^{1-\rho} z(t)\right|=+\infty
$$

and the equation can be solved on $[0, \tilde{T}], \forall \tilde{T}<\hat{T}$. Of course $\hat{T}>T$. This will be apparent from the proof.

Proof of Theorem 3.1

STEP 1:

By definition of $T$, we have

$$
c \sigma(T) \leq 1
$$

We define a map from $C^{0}\left([0, T] ; D\left(A^{1-\rho}\right)\right)$ into itself as follows

$$
\zeta(t)=\mathcal{T}(\eta)(t)=-\int_{0}^{t} e^{-A(t-s)} B(\eta(s)+\phi(s)) \mathrm{d} s .
$$

Majorizing the norm of the integral, and using the properties (2.6), (2.14) we obtain the estimate

$$
\sup _{0 \leq t \leq T}\left|A^{1-\rho} \zeta(t)\right| \leq \frac{c}{2} \frac{T^{\rho}}{\rho} \sup _{0 \leq t \leq T}\left|A^{1-\rho} \eta(t)\right|^{2}+\frac{c}{2} \frac{T^{\rho}}{\rho} \sup _{0 \leq t \leq T}\left|A^{1-\rho} \phi(t)\right|^{2}
$$

with a convenient definition of the constant $c$.

Set

$$
\mathcal{M}^{T}=\left\{\eta \in C^{0}\left([0, T] ; D\left(A^{1-\rho}\right)\right), \eta(0)=0, \quad \sup _{0 \leq t \leq T}\left|A^{1-\rho} \eta(t)\right| \leq c \frac{T^{\rho}}{\rho} \sup _{0 \leq t \leq T}\left|A^{1-\rho} \phi(t)\right|^{2}\right\}
$$

Then, it is easy to check that $\mathcal{T}$ maps $\mathcal{M}^{T}$ into itself. We can proceed with the iteration

$$
z_{n+1}(t)=-\int_{0}^{t} e^{-A(t-s)} B\left(z_{n}(s)+\phi(s)\right) \mathrm{d} s
$$


starting with $z_{0}(t)=0$. By construction

and also

$$
\begin{aligned}
\sup _{0 \leq t \leq T}\left|A^{1-\rho} z_{n}(t)\right| & \leq c \frac{T^{\rho}}{\rho} \sup _{0 \leq t \leq T}\left|A^{1-\rho} \phi(t)\right|^{2} \\
& \leq \frac{c \rho}{T^{\rho}}
\end{aligned}
$$

$$
\left|A^{1-\rho}\left(z_{n+2}(t)-z_{n+1}(t)\right)\right| \leq c \frac{\rho}{T^{\rho}} \int_{0}^{t} \frac{\left|A^{1-\rho}\left(z_{n+1}(s)-z_{n}(s)\right)\right|}{(t-s)^{1-\rho}} \mathrm{d} s .
$$

From these estimates, it follows, as well known, that

$$
z_{n} \rightarrow z \text { in } C^{0}\left([0, T] ; D\left(A^{1-\rho}\right)\right) .
$$

The function $z$ is the unique solution of

$$
z(t)=-\int_{0}^{t} e^{-A(t-s)} B(z(s)+\phi(s)) \mathrm{d} s .
$$

STEP 2:

We prove here that

$$
z(t) \in C^{\delta}\left([0, T] ; D\left(A^{1-\rho}\right)\right), \delta<\rho .
$$

Indeed, the function $B(z(t)+\phi(t))$ belongs to $C^{0}\left([0, T] ; D\left(A^{1-\rho}\right)\right)$, so we can rely on Proposition 2.2, to obtain the result.

\section{STEP 3:}

From Step 2 , and the properties of $B$, it follows, taking account of the assumption on $\phi$, that

$$
B(z+\phi) \in C^{\lambda}([0, T] ; H), \quad \lambda=\min (\beta, \delta), \forall \delta<\rho .
$$

Using then Proposition 2.1, we deduce that

$$
z \in C^{1}([0, T] ; H) \cap C^{0}([0, T] ; D(A))
$$

and also

$$
z \in C^{\rho}\left([0, T] ; D\left(A^{1-\rho}\right)\right)
$$

The proof has been completed.

Continuation Argument:

Suppose $T<T_{0}$, then we can extend a little bit the interval in which one can solve (3.3). Indeed, recall first that

$$
\sup _{0 \leq t \leq T}\left|A^{1-\rho} z(t)\right| \leq c \frac{\rho}{T^{\rho}}
$$

We can write

$$
z(T+\tau)=e^{-A \tau} z(T)+z_{1}(\tau)
$$


and $z_{1}$ is the solution of

$$
z_{1}^{\prime}+A z_{1}+B\left(z_{1}+\phi_{1}\right)=0, \quad z_{1}(0)=0
$$

with

$$
\phi_{1}(\tau)=e^{-A \tau} z(T)+\phi(T+\tau) .
$$

So we get the same equation, with a new function $\phi$. It follows that we can define $z_{1}(\tau)$ on an interval $[0, \theta]$ such that

$$
c \frac{\theta^{\rho}}{\rho}\left[\frac{\rho}{T^{\rho}}+\sup _{0 \leq \tau \leq \theta}\left|A^{1-\rho} \phi(T+\tau)\right|\right] \leq 1
$$

We can proceed in the same way, defining successively smaller and smaller intervals, in which the equation can be extended. The process can be continued as long as $\left|A^{1-\rho} z(t)\right|$ can be bounded.

\section{LOCAL SOLUTiOn OF A FUNCTIONAL PARABOLIC EQUATION}

\subsection{Setting of the problem and statement of results}

We introduce now a functional dependence in the equation $(3.3)$ : replace $\phi(t)$ by $\phi(t ; y)$ as follows

$$
\begin{aligned}
& \phi(t ; \eta): C^{0}\left([0, T] ; D\left(A^{1-\rho}\right)\right) \rightarrow C^{0}\left([0, T] ; D\left(A^{1-\rho}\right)\right) \\
& \phi(t ; \eta): C^{\beta}\left([0, T] ; D\left(A^{1-\rho}\right)\right) \rightarrow C^{\beta}\left([0, T] ; D\left(A^{1-\rho}\right)\right), \beta<\rho
\end{aligned}
$$

with the assumptions

$$
\begin{gathered}
\left|A^{1-\rho}\left(\phi\left(t ; \eta_{1}\right)-\phi\left(t ; \eta_{2}\right)\right)\right| \leq k(t) \sup _{0 \leq s \leq t}\left|A^{1-\rho}\left(\eta_{1}(s)-\eta_{2}(s)\right)\right| \\
\| \phi\left(. ; \eta\left\|_{C^{\beta}\left([0, T] ; D\left(A^{1-\rho}\right)\right)} \leq K(T)+K(T)\right\| \eta\left\|_{C^{0}\left([0, T] ; D\left(A^{1-\rho}\right)\right)}+k(T)\right\| \eta \|_{C^{\beta}\left([0, T] ; D\left(A^{1-\rho}\right)\right)}\right.
\end{gathered}
$$

in which

$$
k(t) \text { increasing, } k(0)=0, k(t) \rightarrow 0, \text { as } t \rightarrow 0 \text {. }
$$

We also assume

$$
\phi(0 ; \eta)=y_{0} \in D\left(A^{1-\beta}\right), \forall \eta
$$

Note that it follows from $(4.2),(4.3),(4.4)$, that

$$
\|\phi(. ; \eta)\|_{C^{0}\left([0, T] ; D\left(A^{1-\rho}\right)\right)} \leq K(T)+k(T)\|\eta\|_{C^{0}\left([0, T] ; D\left(A^{1-\rho}\right)\right)} .
$$

The problem we are going to look at is formulated as follows

$$
\begin{aligned}
& y(t)=z(t)+\phi(t ; y) \\
& z^{\prime}+A z+B(y)=0, \quad z(0)=0
\end{aligned}
$$

and we look for solutions with the regularity

$$
z \in C^{1}([0, T] ; H) \cap C^{0}([0, T] ; D(A)), \quad y \in C^{\beta}\left([0, T] ; D\left(A^{1-\rho}\right)\right) .
$$

We shall prove the following 
Theorem 4.1. Assume (4.1-4.5), and that $T$ satisfies

$$
\begin{aligned}
& c \frac{T^{\rho}}{\rho}(K(T)+1)^{2}+k(T)(K(T)+1) \leq 1 \\
& c \frac{T^{\beta}}{\beta}(K(T)+1)^{2}+k(T)(K(T)+1) \leq 1
\end{aligned}
$$

then there exists one and only one solution of (4.7).

Proof of Theorem 4.1

STEP 1:

We prove uniqueness. Note that from (4.9), one has

$$
k(T)<1
$$

Consider two solutions $y^{1}, z^{1}$ and $y^{2}, z^{2}$. Set

$$
\tilde{y}=y^{1}-y^{2}, \quad \tilde{z}=z^{1}-z^{2} .
$$

We have

$$
\begin{gathered}
\tilde{z}(t)=\int_{0}^{t} e^{-A(t-s)}\left(B\left(y^{1}(s)\right)-B\left(y^{2}(s)\right)\right) \mathrm{d} s \\
\tilde{y}(t)=\tilde{z}(t)+\phi\left(t ; y^{1}\right)-\phi\left(t ; y^{2}\right) .
\end{gathered}
$$

Setting

$$
\theta(t)=\sup _{0 \leq s \leq t}|\tilde{y}(s)|
$$

we deduce easily the estimate

$$
(1-k(T)) \theta(t) \leq C \int_{0}^{t} \theta(s) \mathrm{d} s
$$

and thus the uniqueness follows.

\section{STEP 2:}

We define a map from $C^{\beta}\left([0, T] ; D\left(A^{1-\rho}\right)\right)$ into itself as follows. Pick $\eta \in C^{\beta}\left([0, T] ; D\left(A^{1-\rho}\right)\right)$ such that

$$
\sup _{0 \leq t \leq T}\left|A^{1-\rho} \eta(t)\right| \leq K(T)+1
$$

then it follows from (4.6) that

$$
\sup _{0 \leq t \leq T}\left|A^{1-\rho} \phi(t ; \eta)\right| \leq K(T)+k(T)(K(T)+1) .
$$

We next have from (4.9)

$$
c \frac{T^{\rho}}{\rho}(K(T)+k(T)(K(T)+1)) \leq c \frac{T^{\rho}}{\rho}(K(T)+1)^{2} \leq 1
$$

and thus also

$$
c \frac{T^{\rho}}{\rho} \sup _{0 \leq t \leq T}\left|A^{1-\rho} \phi(t ; \eta)\right| \leq 1
$$


Therefore we can apply Theorem (3.1) to assert that there exists a unique solution

$$
\chi \in C^{1}([0, T] ; H) \cap C^{0}([0, T] ; D(A)) \cap C^{\rho}\left([0, T] ; D\left(A^{1-\rho}\right)\right)
$$

of

We then set

$$
\chi^{\prime}+A \chi+B(\chi+\phi(t ; \eta))=0, \quad \chi(0)=0
$$

$$
\zeta(t)=\chi(t)+\phi(t ; \eta)
$$

which belongs to $C^{\beta}\left([0, T] ; D\left(A^{1-\rho}\right)\right)$. The map is the following

$$
\mathcal{T}(\eta)=\zeta
$$

From Theorem 3.1, we can assert the following estimate

$$
\sup _{0 \leq t \leq T}\left|A^{1-\rho} \chi(t)\right| \leq c \frac{T^{\rho}}{\rho} \sup _{0 \leq t \leq T}\left|A^{1-\rho} \phi(t ; \eta)\right|^{2} .
$$

Recalling previous estimates, it is easy to convince oneself, that thanks to the choice of $T$, one has

$$
\sup _{0 \leq t \leq T}\left|A^{1-\rho} \zeta(t)\right| \leq K(T)+1 .
$$

Thus considering the set

$$
\begin{gathered}
\mathcal{M}^{T}=\left\{\eta \in C^{\rho}\left([0, T] ; D\left(A^{1-\rho}\right)\right) \mid \eta(0)=y_{0},\right. \\
\left.\sup _{0 \leq t \leq T}\left|A^{1-\rho} \eta(t)\right| \leq K(T)+1\right\}
\end{gathered}
$$

we see that $\mathcal{T}$ maps $\mathcal{M}^{T}$ into itself.

\section{STEP 3:}

Consider the iteration

$$
y^{k+1}(t)=\mathcal{T}\left(y^{k}\right)(t)
$$

or more explicitly

$$
\begin{aligned}
& y^{k+1}(t)=z^{k+1}(t)+\phi\left(t ; y^{k}\right) \\
& \left(z^{k+1}\right)^{\prime}+A z^{k+1}+B\left(y^{k+1}\right)=0, \quad z^{k+1}(0)=0
\end{aligned}
$$

with

$$
y^{0}(t)=y_{0}
$$

We deduce

$$
\begin{aligned}
\left|A^{1-\rho}\left(z^{k+2}(t)-z^{k+1}(t)\right)\right| \leq c(K(T)+1) \int_{0}^{t} \frac{\left|A^{1-\rho}\left(z^{k+2}(s)-z^{k+1}(s)\right)\right|}{(t-s)^{1-\rho}} \mathrm{d} s \\
+c(K(T)+1) \int_{0}^{t} \frac{\left|A^{1-\rho}\left(\phi\left(s ; y^{k+1}\right)-\phi\left(s ; y^{k}\right)\right)\right|}{(t-s)^{1-\rho}} \mathrm{d} s .
\end{aligned}
$$


So one is led to an inequality of the form

$$
\theta(t) \leq a \int_{0}^{t} \frac{\theta(s)}{(t-s)^{1-\rho}} \mathrm{d} s+a \int_{0}^{t} \frac{g(s)}{(t-s)^{1-\rho}} \mathrm{d} s
$$

which implies

$$
\theta(t) \leq K^{\prime}(T) \int_{0}^{t} \frac{g(s)}{(t-s)^{1-\rho}} \mathrm{d} s
$$

Therefore we can write

$$
\left|A^{1-\rho}\left(z^{k+2}(t)-z^{k+1}(t)\right)\right| \leq K^{\prime}(T) \int_{0}^{t} \frac{\left|A^{1-\rho}\left(\phi\left(s ; y^{k+1}\right)-\phi\left(s ; y^{k}\right)\right)\right|}{(t-s)^{1-\rho}} \mathrm{d} s
$$

and thus also

$$
\left|A^{1-\rho}\left(y^{k+2}(t)-y^{k+1}(t)\right)\right| \leq\left|A^{1-\rho}\left(\phi\left(t ; y^{k+1}\right)-\phi\left(t ; y^{k}\right)\right)\right|+K^{\prime}(T) \int_{0}^{t} \frac{\left|A^{1-\rho}\left(\phi\left(s ; y^{k+1}\right)-\phi\left(s ; y^{k}\right)\right)\right|}{(t-s)^{1-\rho}} \mathrm{d} s .
$$

Using the assumption (4.2), and setting

$$
\phi^{k}(t)=\sup _{0 \leq s \leq t}\left|A^{1-\rho}\left(y^{k+1}(s)-y^{k}(s)\right)\right|
$$

it easily follows from (4.17) the inequality

$$
\phi^{k+1}(t) \leq k(T)\left[\phi^{k}(t)+K^{\prime}(T) \int_{0}^{t} \frac{\phi^{k}(s)}{(t-s)^{1-\rho}} \mathrm{d} s\right] .
$$

An iteration shows that

$$
\phi^{k}(t) \leq(k(T))^{k} K^{\prime \prime}(T)
$$

Thus

$$
\sup _{0 \leq s \leq T}\left|A^{1-\rho}\left(y^{k+1}(s)-y^{k}(s)\right)\right| \leq(k(T))^{k} K^{\prime \prime}(T) .
$$

Since $k(T)<1$, the series converges and thus

$$
y^{k} \rightarrow y \text { in } C^{0}\left([0, T] ; D\left(A^{1-\rho}\right)\right) .
$$

\section{STEP 4:}

We prove here an estimate with respect to the Hölder norm. Going back to the functions $\chi, \zeta$ associated with $\eta$, and recalling the estimate (2.8) in Proposition 2.1 and Corollary 2.1, we can assert that

$$
\|\chi\|_{C^{\rho}\left([0, T] ; D\left(A^{1-\rho}\right)\right)} \leq c\|B(\zeta)\|_{C^{0}([0, T] ; H)}+c \frac{T^{\beta}}{\beta}\|B(\zeta)\|_{C^{\beta}([0, T] ; H)}
$$

therefore also

$$
\|\chi\|_{C^{\rho}\left([0, T] ; D\left(A^{1-\rho}\right)\right)} \leq c\|\zeta\|_{C^{0}\left([0, T] ; D\left(A^{1-\rho}\right)\right)}^{2}+c \frac{T^{\beta}}{\beta}\|\zeta\|_{C^{\beta}\left([0, T] ; D\left(A^{1-\rho}\right)\right)}\|\zeta\|_{C^{0}\left([0, T] ; D\left(A^{1-\rho}\right)\right)} .
$$


We can replace in the left hand side $\|\chi\|_{C^{\rho}\left([0, T] ; D\left(A^{1-\rho}\right)\right)}$ by $\|\chi\|_{C^{\beta}\left([0, T] ; D\left(A^{1-\rho}\right)\right)}$.

Finally, using (4.3) we deduce

$$
\begin{aligned}
\|\zeta\|_{C^{\beta}\left([0, T] ; D\left(A^{1-\rho}\right)\right)} \leq & K(T)+K(T)\|\eta\|_{C^{0}\left([0, T] ; D\left(A^{1-\rho}\right)\right)}+k(T)\|\eta\|_{C^{\beta}\left([0, T] ; D\left(A^{1-\rho}\right)\right)}+c\|\zeta\|_{C^{0}\left([0, T] ; D\left(A^{1-\rho}\right)\right)}^{2} \\
& +c \frac{T^{\beta}}{\beta}\|\zeta\|_{C^{\beta}\left([0, T] ; D\left(A^{1-\rho}\right)\right)}\|\zeta\|_{C^{0}\left([0, T] ; D\left(A^{1-\rho}\right)\right)} .
\end{aligned}
$$

We know that hence from (4.21), it follows

$$
\|\eta\|_{C^{0}\left([0, T] ; D\left(A^{1-\rho}\right)\right)} \leq K(T)+1 \Rightarrow\|\zeta\|_{C^{0}\left([0, T] ; D\left(A^{1-\rho}\right)\right)} \leq K(T)+1
$$

$$
\begin{aligned}
\|\zeta\|_{C^{\beta}\left([0, T] ; D\left(A^{1-\rho}\right)\right) \leq} & K(T)+K(T)(K(T)+1)+k(T)\|\eta\|_{C^{\beta}\left([0, T] ; D\left(A^{1-\rho}\right)\right)} \\
& +c(K(T)+1)^{2}+c \frac{T^{\beta}}{\beta}\|\zeta\|_{C^{\beta}\left([0, T] ; D\left(A^{1-\rho}\right)\right)}(K(T)+1) .
\end{aligned}
$$

Therefore we arrive at

$$
\left(1-c \frac{T^{\beta}}{\beta}(K(T)+1)\right)\|\zeta\|_{C^{\beta}\left([0, T] ; D\left(A^{1-\rho}\right)\right)} \leq K^{\prime}(T)+k(T)\|\eta\|_{C^{\beta}\left([0, T] ; D\left(A^{1-\rho}\right)\right)}
$$

or

where in particular

$$
\|\zeta\|_{C^{\beta}\left([0, T] ; D\left(A^{1-\rho}\right)\right)} \leq K^{\prime \prime}(T)+k^{\prime}(T)\|\eta\|_{C^{\beta}\left([0, T] ; D\left(A^{1-\rho}\right)\right)}
$$

$$
k^{\prime}(T)=\frac{k(T)}{\left(1-c \frac{T^{\beta}}{\beta}(K(T)+1)\right)} .
$$

From (4.9), we have $k^{\prime}(T)<1$. Applying this relation to the sequence $y^{k}$, it follows

$$
\left\|y^{k+1}\right\|_{C^{\beta}\left([0, T] ; D\left(A^{1-\rho}\right)\right)} \leq K^{\prime \prime}(T)+k^{\prime}(T)\left\|y^{k}\right\|_{C^{\beta}\left([0, T] ; D\left(A^{1-\rho}\right)\right)}
$$

from which it follows that

$$
\left\|y^{k}\right\|_{C^{\beta}\left([0, T] ; D\left(A^{1-\rho}\right)\right)} \leq C .
$$

Therefore the limit $y$ belongs also to $C^{\beta}\left([0, T] ; D\left(A^{1-\rho}\right)\right)$. The proof has been completed.

\section{Stochastic equations}

\subsection{Model with external noise}

We consider a probability space $\Omega, \mathcal{A}, P$, a filtration $\mathcal{F}^{t}$ and an $\mathcal{F}^{t} m$-dimensional Wiener process $w(t)$. Let $g(t)$ be an adapted stochastic process, with values in $\left(D\left(A^{\rho^{\prime}}\right)\right)^{*}$ such that

$$
A^{-\rho^{\prime}} g(.) \in L^{q}([0, T] ; H), \text { a.s. }, 0 \leq \rho^{\prime}, \rho-\rho^{\prime}>\frac{1}{q} .
$$

Consider next a stochastic process $G(t)$, with values in $\left(D\left(A^{\varepsilon^{\prime}}\right)\right)^{m}$, such that

$$
A^{\varepsilon^{\prime}} G(.) \in L^{2 r}\left(\Omega, \mathcal{A}, P ; L^{\infty}(0, T ; H)\right), \quad \rho+\varepsilon^{\prime}>\frac{1}{2 r}+\frac{1}{2} .
$$

Let finally

$$
y_{0}, \mathcal{F}^{0} \text { measurable with values in } D\left(A^{1-\rho_{0}}\right), \quad \rho_{0}<\rho .
$$


We then define the stochastic process

$$
\phi(t)=e^{-A t} y_{0}+\int_{0}^{t} e^{-A(t-s)} g(s) \mathrm{d} s+\int_{0}^{t} e^{-A(t-s)} G(s) \mathrm{d} w .
$$

We state the following

Proposition 5.1. With the assumptions (5.1-5.3), the process $\phi(t)$ has a.s. trajectories in $C^{\beta}\left([0, T] ; D\left(A^{1-\rho}\right)\right)$, with

$$
\beta \leq \rho-\rho_{0}, \quad \beta<\min \left(\rho-\rho^{\prime}-\frac{1}{q}, \rho+\varepsilon^{\prime}-\frac{1}{2 r}-\frac{1}{2}\right)
$$

Proof.

STEP 1:

Writing

it follows

$$
A^{1-\rho}\left(e^{-A(t+h)}-e^{-A t}\right) y_{0}=-h^{\rho-\rho_{0}}\left(\int_{0}^{1} h^{1-\rho+\rho_{0}} A^{1-\rho+\rho_{0}} e^{-A h \tau} \mathrm{d} \tau\right) A^{1-\rho_{0}} e^{-t A} y_{0}
$$

hence

$$
\left|A^{1-\rho}\left(e^{-A(t+h)}-e^{-A t}\right) y_{0}\right| \leq c h^{\rho-\rho_{0}}\left|A^{1-\rho_{0}} y_{0}\right|
$$

$\underline{\text { STEP 2: }}$

$$
e^{-A t} y_{0} \in C^{\rho-\rho_{0}}\left([0, T] ; D\left(A^{1-\rho}\right)\right)
$$

Set

then

$$
X(t)=\int_{0}^{t} e^{-A(t-s)} g(s) \mathrm{d} s
$$

$$
A^{1-\rho}(X(t+h)-X(t))=A^{1-\rho} \int_{t}^{t+h} e^{-A(t+h-s)} g(s) \mathrm{d} s+A^{1-\rho}\left(e^{-A h}-I\right) X(t)
$$

Write

$$
\begin{aligned}
\left|A^{1-\rho} \int_{t}^{t+h} e^{-A(t+h-s)} g(s) \mathrm{d} s\right| & =\left|\int_{t}^{t+h} A^{1-\rho+\rho^{\prime}} e^{-A(t+h-s)} A^{-\rho^{\prime}} g(s) \mathrm{d} s\right| \\
& \leq c \int_{t}^{t+h} \frac{\left|A^{-\rho^{\prime}} g(s)\right|}{(t+h-s)^{1-\rho+\rho^{\prime}}} \mathrm{d} s
\end{aligned}
$$

and from Hölder's inequality we get

$$
\leq \operatorname{ch}^{\rho-\rho^{\prime}-\frac{1}{q}}\|g\|_{L^{q}([0, T] ; H)} .
$$

Turning to the second integral, we pick any $\beta<\rho-\rho^{\prime}-\frac{1}{q}$, and write

$$
\begin{aligned}
\left|A^{1-\rho}\left(e^{-A h}-I\right) X(t)\right| & =h^{\beta}\left|\int_{0}^{1} h^{1-\beta} A^{1-\beta} e^{-A h \tau} \mathrm{d} \tau \int_{0}^{t} A^{1-\rho+\rho^{\prime}+\beta} e^{-A(t-s)} A^{-\rho^{\prime}} g(s) \mathrm{d} s\right| \\
& \leq c h^{\beta} \int_{0}^{t} \frac{\left|A^{-\rho^{\prime}} g(s)\right|}{(t-s)^{1-\rho+\rho^{\prime}+\beta}} \mathrm{d} s .
\end{aligned}
$$

Using again Hölder's inequality we get

$$
\left|A^{1-\rho}\left(e^{-A h}-I\right) X(t)\right| \leq c h^{\beta}\|g\|_{L^{q}([0, T] ; H)} .
$$


So $X(t)$ is a.s. Hölder with exponent $\beta<\rho-\rho^{\prime}-\frac{1}{q}$.

$\underline{\text { STEP 3: }}$

Set

$$
Y(t)=\int_{0}^{t} A^{1-\rho} e^{-A(t-s)} G(s) \mathrm{d} w .
$$

Then, we are in the framework of Appendix 1, with

$$
\Phi(s)=A^{1-\rho} G(s)
$$

and

$$
A^{-\beta} \Phi(s)=A^{1-\rho-\beta} G(s)=A^{\varepsilon^{\prime}} G(s)
$$

with $\beta=1-\rho-\varepsilon^{\prime}$. Applying Theorem 7.1 of Appendix 1, we get immediately that $Y(t)$ is a.s. Hölder with exponent strictly smaller than $\rho+\varepsilon^{\prime}-\frac{1}{2}-\frac{1}{2 r}$.

The proof has been completed.

It is thus possible to take in equation (3.3), the stochastic process $\phi(t)$. Then

$$
y(t)=z(t)+\phi(t)
$$

is the mild solution of the stochastic equation

$$
\mathrm{d} y+(A y+B(y)) \mathrm{d} t=g(t) \mathrm{d} t+G(t) d w, \quad y(0)=y_{0}
$$

and is defined as an adapted process, with trajectories in $C^{\beta}\left([0, T] ; D\left(A^{1-\rho}\right)\right)$, with $\beta$ as in (5.5). We can take as minimal interval of definition the stopping time $T$ such that

$$
T=\inf \left\{t\left|c \frac{t^{\rho}}{\rho} \sup _{0 \leq s \leq t}\right| A^{1-\rho} \phi(s) \mid \geq 1\right\}
$$

provided, of course, that the assumptions (5.1),(5.2)hold for any compact interval.

\subsection{Model with smooth functional dependence}

We introduce now a stochastic process $\phi(t)$ with functional dependence, namely

$$
\phi(t ; \eta)=e^{-A t} y_{0}+\int_{0}^{t} e^{-A(t-s)} g(\eta)(s) \mathrm{d} s+\int_{0}^{t} e^{-A(t-s)} G(\eta)(s) \mathrm{d} w .
$$

The functional dependence is assumed to be smooth, which means that we are able to perform an integration by part in the stochastic integral, so that we have, in fact

$$
\phi(t ; \eta)=e^{-A t} y_{0}+\int_{0}^{t} e^{-A(t-s)} \Gamma(\eta)(s) \cdot w(s) \mathrm{d} s+G(\eta)(t) \cdot w(t)
$$

with the definition

$$
\Gamma(\eta)(t)=g(\eta)(t)-A G(\eta)(t)-G(\eta)^{\prime}(t)
$$


with the assumptions

$$
\begin{aligned}
\sup _{0 \leq t \leq T}\left|A^{1-\varepsilon} G(\eta)^{\prime}(t)\right|+\left|A^{1-\varepsilon} G(\eta)(0)\right| & \leq K+K \sup _{0 \leq t \leq T}\left|A^{1-\rho} \eta(t)\right|, \quad \varepsilon<\rho \\
\left|A^{-\varepsilon}\left(\Gamma\left(\eta_{1}\right)(t)-\Gamma\left(\eta_{2}\right)(t)\right)\right| & \leq K \sup _{0 \leq s \leq t}\left|A^{1-\rho}\left(\eta_{1}(s)-\eta_{2}(s)\right)\right| \\
\left|A^{1-\rho}\left(G\left(\eta_{1}\right)(t)-G\left(\eta_{2}\right)(t)\right)\right| & \leq K \sup _{0 \leq s \leq t}\left|A^{1-\rho}\left(\eta_{1}(s)-\eta_{2}(s)\right)\right| \\
\sup _{0 \leq t \leq T}\left|A^{-\varepsilon} g(0)(t)\right| & \leq K .
\end{aligned}
$$

The constants may depend on $\varepsilon, \beta$, but not on $T$. Set

$$
\beta<\rho-\varepsilon
$$

and assume

$$
y_{0} \in D\left(A^{1-\rho+\beta}\right) .
$$

We then state the

Lemma 5.1. We have the following consequences of assumptions (5.11-5.14)

$$
\begin{aligned}
& \sup _{0 \leq t \leq T}\left|A^{1-\varepsilon} G(\eta)(t)\right| \leq K+K \sup _{0 \leq t \leq T}\left|A^{1-\rho} \eta(t)\right| \\
& \sup _{0 \leq t \leq T}\left|A^{-\varepsilon} \Gamma(\eta)(t)\right| \leq K+K \sup _{0 \leq t \leq T}\left|A^{1-\rho} \eta(t)\right| \\
& \sup _{0 \leq t \leq T}\left|A^{-\varepsilon} g(\eta)(t)\right| \leq K+K \sup _{0 \leq t \leq T}\left|A^{1-\rho} \eta(t)\right| .
\end{aligned}
$$

Proof.

Since

$$
G(\eta)(t)=G(\eta)(0)+\int_{0}^{t} G(\eta)^{\prime}(s) \mathrm{d} s
$$

then (5.17) is an obvious consequence of (5.11). Since $\rho>\varepsilon$, we also have

$$
\sup _{0 \leq t \leq T}\left|A^{1-\rho} G(\eta)(t)\right| \leq K+K \sup _{0 \leq t \leq T}\left|A^{1-\rho} \eta(t)\right| .
$$

Now

and using (5.14), (5.17), (5.11), we have

$$
A^{-\varepsilon} \Gamma(0)(t)=A^{-\varepsilon} g(0)(t)-A^{1-\varepsilon} G(0)(t)-A^{-\varepsilon} G(0)^{\prime}(t)
$$

$$
\sup _{0 \leq t \leq T}\left|A^{-\varepsilon} \Gamma(0)(t)\right| \leq K
$$

which together with assumption (5.12) implies $(5.18)$. Using $(5.17,5.18)$ with $(5.11)$, we obtain $(5.19)$. The proof has been completed.

Lemma 5.2. For the the process $\phi(t ; \eta)$ defined by (5.9) the assumptions (4.2), (4.3) are satisfied with the following values of $k(t), K(T)$

$$
k(t)=K^{\prime}\left(1+t^{\rho-\varepsilon}\right) \sup _{0 \leq s \leq t}|w(s)|
$$




$$
K(T)=c\left\|y_{0}\right\|_{D\left(A^{1-\rho+\beta}\right)}+K^{\prime}\|w\|_{C^{\beta}\left([0, T] ; R^{m}\right)}+K^{\prime}\left[T^{\rho-\varepsilon-\beta}+T^{1-\beta}\right]\|w\|_{C^{0}\left([0, T] ; R^{m}\right)}
$$

where $c, K^{\prime}$ are constants, with $K^{\prime}$ depending on $\beta, \rho, \varepsilon$, but not on $T$.

Proof. Let

$$
\psi(t)=\int_{0}^{t} e^{-A(t-s)} \Gamma(\eta)(s) \cdot w(s) \mathrm{d} s
$$

then from the definition (5.9) we can write

$$
\|\phi(. ; \eta)\|_{C^{\beta}\left([0, T] ; D\left(A^{1-\rho}\right)\right)} \leq\left\|e^{-A \cdot} y_{0}\right\|_{C^{\beta}\left([0, T] ; D\left(A^{1-\rho}\right)\right)}+\|\psi\|_{C^{\beta}\left([0, T] ; D\left(A^{1-\rho}\right)\right)}+\|G(\eta)(.) w(.)\|_{C^{\beta}\left([0, T] ; D\left(A^{1-\rho}\right)\right)} .
$$

The first term is estimated by $c\left\|y_{0}\right\|_{D\left(A^{1-\rho+\beta}\right)}$. To estimate the second one, we rely on Proposition 2.2 , see (2.10), applied with

$$
g=A^{-\varepsilon} \Gamma(\eta) \cdot w, \quad \delta=\beta
$$

and $\rho$ changed into $\rho-\varepsilon$. We can then assert that

$$
\|\psi\|_{C^{\beta}\left([0, T] ; D\left(A^{1-\rho}\right)\right)} \leq c\left\|A^{-\varepsilon} \Gamma(\eta) \cdot w\right\|_{C^{0}([0, T] ; H)} T^{\rho-\varepsilon-\beta}
$$

where $c$ depends here on $\beta, \rho, \varepsilon$. Hence

$$
\|\psi\|_{C^{\beta}\left([0, T] ; D\left(A^{1-\rho}\right)\right)} \leq c\left\|A^{-\varepsilon} \Gamma(\eta)\right\|_{C^{0}\left([0, T] ; H^{m}\right)}\|w\|_{C^{0}\left([0, T] ; R^{m}\right)} T^{\rho-\varepsilon-\beta} .
$$

Next

$$
\begin{aligned}
\|G(\eta)(.) w(.)\|_{C^{\beta}\left([0, T] ; D\left(A^{1-\rho}\right)\right) \leq} & \|G(\eta)\|_{C^{0}\left([0, T] ; D\left(A^{1-\rho}\right)\right)}\|w\|_{C^{\beta}\left([0, T] ; R^{m}\right)} \\
& +\|G(\eta)\|_{C^{\beta}\left([0, T] ; D\left(A^{1-\rho}\right)\right)}\|w\|_{C^{0}\left([0, T] ; R^{m}\right)} .
\end{aligned}
$$

But, as easily seen

$$
\|G(\eta)\|_{C^{\beta}\left([0, T] ; D\left(A^{1-\rho}\right)\right)} \leq c T^{1-\beta}\left\|G^{\prime}(\eta)\right\|_{C^{0}\left([0, T] ; D\left(A^{1-\rho}\right)\right)} .
$$

Collecting results, and recalling (5.11) and Lemma 5.1, we obtain the value of the constant $K(T)$, to verify the assumption (4.3). In fact, there is no term involving explicitly $\|\eta\|_{C^{\beta}\left([0, T] ; D\left(A^{1-\rho}\right)\right)}$.

Let us now check (4.2). It is an easy consequence of the assumptions (5.12), (5.13). We obtain the value (5.21) for $k(t)$. The properties of the Wiener process imply that a.s. $k(t)$ is increasing, vanishes at 0 and tends to 0 as $t \rightarrow 0$.

The proof has been completed. So we can state the following:

Theorem 5.1. We assume (5.11-5.14). Then there exists one and only one mild solution of the stochastic Navier Stokes equation

$$
\mathrm{d} y+(A y+B(y)) \mathrm{d} t=g(y)(t) \mathrm{d} t+G(y)(t) \mathrm{d} w(t), \quad y(0)=y_{0}
$$

on the interval $[0, T]$, where $T$ is the random time defined by the first condition (4.9), where $k(T), K(T)$ are defined by formulas (5.21), (5.22). The trajectories of the process $y$ belong a.s. to $C^{\beta}\left([0, T] ; D\left(A^{1-\rho}\right)\right)$ with $\beta<\rho-\varepsilon$.

Proof.

It is a direct application of Theorem 4.1. A mild solution is a solution of the integral equation

$$
y(t)=e^{-A t} y_{0}-\int_{0}^{t} e^{-A(t-s)} B(y(s)) \mathrm{d} s+\int_{0}^{t} e^{-A(t-s)}(g(y)(s) \mathrm{d} s+G(y)(s) \mathrm{d} w(s)) .
$$


Setting

$$
\phi(t ; y)=e^{-A t} y_{0}+\int_{0}^{t} e^{-A(t-s)}(g(y)(s) \mathrm{d} s+G(y)(s) \mathrm{d} w(s))
$$

and considering

$$
z(t)=y(t)-\phi(t ; y)
$$

we are in the framework of Theorem 4.1. Thanks to Lemma 5.2, all assumptions are satisfied. We do not need to use the second condition (4.9), because there is no dependence in $\|\eta\|_{C^{\beta}\left([0, T] ; D\left(A^{1-\rho}\right)\right)}$, in the condition (4.3). The proof has been completed.

Example

Suppose we take

$$
\begin{gathered}
g(\eta)(t)=g(\eta(t)) \\
G(\eta)(t)=\frac{1}{\alpha} \int_{0}^{t} e^{-\frac{A}{\alpha}(t-s)} \sigma(\eta(s)) \mathrm{d} s
\end{gathered}
$$

and we assume the following properties

$$
\begin{gathered}
\left|A^{-\varepsilon}\left(g\left(\eta_{1}\right)-g\left(\eta_{2}\right)\right)\right| \leq K\left|A^{1-\rho}\left(\eta_{1}-\eta_{2}\right)\right| \\
\left|A^{1-\varepsilon^{\prime}}\left(\sigma\left(\eta_{1}\right)-\sigma\left(\eta_{2}\right)\right)\right| \leq K\left|A^{1-\rho}\left(\eta_{1}-\eta_{2}\right)\right|, \quad \varepsilon^{\prime}<\varepsilon
\end{gathered}
$$

then the assumptions (5.11-5.14) are all satisfied. In fact, (5.30) is more than necessary. It was made in order to verify (5.11). That is to say, to estimate $A^{1-\varepsilon} G(\eta)^{\prime}(t)$. This last assumption was a compromise in order to estimate at the same time $A^{1-\varepsilon} G(\eta)(t)$, needed to estimate $A^{-\varepsilon} \Gamma(\eta)(t)$, and $A^{1-\rho} G(\eta)^{\prime}(t)$, used to check that that $G(\eta)$ belongs to $C^{\beta}\left([0, T] ; D\left(A^{1-\rho}\right)\right)$. Here, because of the form of $G(\eta)$, in particular thanks to the presence of the operator the semi group $e^{-A t}$ in the integral, we can verify more directly the conditions of applicability of Theorem 4.1 . It is enough to replace (5.30) by

$$
\left|A^{-\varepsilon^{\prime}}\left(\sigma\left(\eta_{1}\right)-\sigma\left(\eta_{2}\right)\right)\right| \leq K\left|A^{1-\rho}\left(\eta_{1}-\eta_{2}\right)\right|, \quad \varepsilon^{\prime}<\varepsilon
$$

and we state the following

Theorem 5.2. We assume (5.27, 5.28, 5.29, 5.31). Then there exists one and only one mild solution of the stochastic Navier Stokes equation

$$
\mathrm{d} y+(A y+B(y)) \mathrm{d} t=g(y(t)) \mathrm{d} t+\left(\frac{1}{\alpha} \int_{0}^{t} e^{-\frac{A}{\alpha}(t-s)} \sigma(y(s)) \mathrm{d} s\right) \mathrm{d} w(t), \quad y(0)=y_{0}
$$

on the interval $[0, T]$, where $T$ is the random time defined by the first condition (4.9), where $k(T), K(T)$ are defined by formulas (5.21, 5.22). The trajectories of the process y belong a.s. to $C^{\beta}\left([0, T] ; D\left(A^{1-\rho}\right)\right)$, with $\beta<\rho-\varepsilon$.

Remark 5.1. The model (5.32) can be seen as taking account of the memory of the state $y(s), s<t$, with a discount factor. It is convenient to use as a discount factor, the semi group related to the operator $A$. As $\alpha \rightarrow 0$, we recover $A^{-1} \sigma(y(t))$. 


\section{Formulation of a local solution}

We formulate in this section the problem of finding a local solution for a stochastic Navier-stokes equation. We consider a process $y(t)$ with values in $D\left(A^{1-\rho}\right)$, such that

$$
\begin{aligned}
y\left(t \wedge \sigma^{M}\right)= & e^{-A t \wedge \sigma^{M}} y_{0}-\int_{0}^{t \wedge \sigma^{M}} e^{-A\left(t \wedge \sigma^{M}-s\right)} B(y(s)) \mathrm{d} s \\
& +\int_{0}^{t \wedge \sigma^{M}} e^{-A\left(t \wedge \sigma^{M}-s\right)} g(y(s)) \mathrm{d} s+\int_{0}^{t \wedge \sigma^{M}} e^{-A\left(t \wedge \sigma^{M}-s\right)} G(y(s)) \mathrm{d} w(s), \quad \forall M
\end{aligned}
$$

where

$$
\sigma^{M}=\inf \left\{0 \leq t \leq T:\left|A^{1-\rho} y(t)\right| \geq N_{0}+M\right\}
$$

We make the following assumptions. There is given an underlying probability system $\Omega, \mathcal{A}, P$, with an $m$ dimensional Wiener process $w(t)$, and let

$$
\mathcal{F}^{t}=\sigma-\text { algebra generated by } w(s), 0 \leq s \leq t .
$$

The operators $A, B$ are of course those of Section 2.1 and (2.12). We assume in addition that

$$
\begin{gathered}
\left|A^{-\rho^{\prime}}(g(u)-g(v))\right| \leq K\left|A^{1-\rho}(u-v)\right|, \quad 0 \leq \rho^{\prime}<\rho \\
\left|A^{\varepsilon^{\prime}}(G(u)-G(v))\right| \leq K\left|A^{1-\rho}(u-v)\right|, \quad 1 \geq \varepsilon^{\prime}+\rho>\frac{1}{2}
\end{gathered}
$$

and

$$
y_{0} \text { is deterministic }\left|A^{1-\rho} y_{0}\right|=N_{0}<\infty \text {. }
$$

Our objective is to prove the following

Theorem 6.1. We assume (6.3, 6.4, 6.5), then for each $T$ we can construct an increasing sequence of stopping times $\sigma^{M} \leq T$, and a process $y(t)$ defined on $[0, \tilde{\sigma})$, where

$$
\tilde{\sigma}=\uparrow \sigma^{M} .
$$

The process $y(t)$ is adapted, is a solution of (6.1,6.2), has a continuous modification with values in $D\left(A^{1-\rho}\right)$, which is Hölder with exponent strictly smaller than $\min \left(\rho-\rho^{\prime}, \varepsilon^{\prime}+\rho-\frac{1}{2}\right)$. The solution is unique in the sense that two solutions $y(t), y^{*}(t)$ verify

$$
\text { a.s. } y(t)=y^{*}(t), \forall t<\tilde{\sigma} \text {. }
$$

Moreover, if $\tilde{\sigma}<T$, then

$$
\left|A^{1-\rho} y(t)\right| \uparrow \infty, \text { as } t \uparrow \tilde{\sigma} \text {. }
$$

Proof.

We begin by constructing the process $y^{1}(t)$ which is the solution on any finite time interval $[0, T]$ of the equation

$$
y^{1}(t)=e^{-A t} y_{0}-\int_{0}^{t} e^{-A(t-s)} B_{N_{0}+1}\left(y^{1}(s)\right) \mathrm{d} s+\int_{0}^{t} e^{-A(t-s)} g\left(y^{1}(s)\right) \mathrm{d} s+\int_{0}^{t} e^{-A(t-s)} G\left(y^{1}(s)\right) \mathrm{d} w(s) .
$$


We use Appendix 2 to solve this equation, see Theorem 8.1, and (8.1) for the definition of $B_{N_{0}+1}$. Note that condition (8.7) holds for $r=+\infty$. So we know that there exists a unique process $y^{1}(t)$, defined on any interval $[0, T]$, which has a continuous modification with values in $D\left(A^{1-\rho}\right)$, which is Hölder with exponent strictly smaller than $\min \left(\rho-\rho^{\prime}, \varepsilon^{\prime}+\rho-\frac{1}{2}\right)$, and satisfies (6.5). We then define the stopping time

$$
\sigma^{1}=\inf \left\{0 \leq t \leq T|| A^{1-\rho} y^{1} \mid \geq N_{0}+1\right\}
$$

One has

$$
\left|A^{1-\rho} y^{1}\left(\sigma^{1}\right)\right| \leq N_{0}+1
$$

and we set

$$
y_{\sigma^{1}}=y^{1}\left(\sigma^{1}\right)
$$

Using again Appendix 2, we look for $y^{2}$ to be the solution of

$$
\begin{aligned}
y^{2}(t)= & E\left[e^{-A\left(t-\sigma^{1}\right)^{+}} y_{\sigma^{1}} \mid \mathcal{F}^{t}\right]-\int_{0}^{t} \Pi_{s \geq \sigma^{1}} e^{-A(t-s)} B_{N_{0}+2}\left(y^{2}(s)\right) \mathrm{d} s \\
& +\int_{0}^{t} \Pi_{s \geq \sigma^{1}} e^{-A(t-s)} g\left(y^{2}(s)\right) \mathrm{d} s+\int_{0}^{t} \mathbb{I}_{s \geq \sigma^{1}} e^{-A(t-s)} G\left(y^{2}(s)\right) \mathrm{d} w(s) .
\end{aligned}
$$

The process $y^{2}$ is well defined on any finite interval of time $[0, T]$, and enjoys all the properties stated for $y^{1}$ except that the continuity and Hölder continuity hold for

$$
y^{2}(t)-E\left[e^{-A\left(t-\sigma^{1}\right)^{+}} y_{\sigma^{1}} \mid \mathcal{F}^{t}\right]
$$

We define the stopping time

$$
\sigma^{2}=\inf \left\{0 \leq t \leq T|| A^{1-\rho} y^{2} \mid \geq N_{0}+2\right\}
$$

and set

$$
y_{\sigma^{2}}=y^{2}\left(\sigma^{2}\right)
$$

In general, suppose we have defined $y^{k}, \sigma^{k}, y_{\sigma^{k}}$, then we define $y^{k+1}$ by solving the equation

$$
\begin{aligned}
y^{k+1}(t)=E\left[e^{-A\left(t-\sigma^{k}\right)^{+}} y_{\sigma^{k}} \mid \mathcal{F}^{t}\right] & -\int_{0}^{t} \mathbb{I}_{s \geq \sigma^{k}} e^{-A(t-s)} B_{N_{0}+k+1}\left(y^{k+1}(s)\right) \mathrm{d} s \\
& +\int_{0}^{t} \mathbb{I}_{s \geq \sigma^{k}} e^{-A(t-s)} g\left(y^{k+1}(s)\right) \mathrm{d} s+\int_{0}^{t} \mathbb{I}_{s \geq \sigma^{k}} e^{-A(t-s)} G\left(y^{k+1}(s)\right) \mathrm{d} w(s)
\end{aligned}
$$

and we set

$$
\begin{aligned}
\sigma^{k+1} & =\inf \left\{0 \leq t \leq T|| A^{1-\rho} y^{k+1}(t) \mid \geq N_{0}+k+1\right\} \\
y_{\sigma^{k+1}} & =y^{k+1}\left(\sigma^{k+1}\right) .
\end{aligned}
$$

We have constructed the sequence of stopping times $\sigma^{k}$. We then construct the process $y(t)$ as follows

$$
y(t)=y^{k+1}(t), \forall t \in\left[\sigma^{k}, \sigma^{k+1}\right] .
$$


Let

$$
\tilde{\sigma}=\uparrow \sigma^{k} .
$$

The process $y(t)$ is defined on $[0, \tilde{\sigma})$. It is a continuous process, since on a switching point $\sigma^{k}$, whenever $\sigma^{k}<T$, one has

$$
y\left(\sigma^{k}\right)=y^{k}\left(\sigma^{k}\right)=y_{\sigma^{k}}=y^{k+1}\left(\sigma^{k}\right) .
$$

It is also Hölder continuous, with the same exponent as the processes $y^{k}$. Clearly, by construction,

$$
\sigma^{k}=\inf \left\{0 \leq t \leq T|| A^{1-\rho} y(t) \mid \geq N_{0}+k\right\}
$$

Let us check that the equation (6.1) is satisfied, with $M=N_{0}+k$. Note that, for $0 \leq s \leq \sigma^{1}$, one has

$$
B_{N_{0}+1}\left(y^{1}(s)\right)=B\left(y^{1}(s)\right)=B(y(s))
$$

and thus one has

$$
\begin{aligned}
y\left(t \wedge \sigma^{1}\right)= & e^{-A t \wedge \sigma^{1}} y_{0}-\int_{0}^{t \wedge \sigma^{1}} e^{-A\left(t \wedge \sigma^{1}-s\right)} B(y(s)) \mathrm{d} s \\
& +\int_{0}^{t \wedge \sigma^{1}} e^{-A\left(t \wedge \sigma^{1}-s\right)} g(y(s)) \mathrm{d} s+\int_{0}^{t \wedge \sigma^{1}} e^{-A\left(t \wedge \sigma^{1}-s\right)} G(y(s)) \mathrm{d} w(s) .
\end{aligned}
$$

In particular,

$$
y\left(\sigma^{1}\right)=y_{\sigma^{1}}=e^{-A \sigma^{1}} y_{0}-\int_{0}^{\sigma^{1}} e^{-A\left(\sigma^{1}-s\right)} B(y(s)) \mathrm{d} s+\int_{0}^{\sigma^{1}} e^{-A\left(\sigma^{1}-s\right)} g(y(s)) \mathrm{d} s+\int_{0}^{\sigma^{1}} e^{-A\left(\sigma^{1}-s\right)} G(y(s)) \mathrm{d} w(s) .
$$

Similarly, one has, for $t \wedge \sigma^{2} \geq \sigma^{1}$,

$$
\begin{aligned}
y\left(t \wedge \sigma^{2}\right)= & e^{-A\left(t \wedge \sigma^{2}-\sigma^{1}\right)} y_{\sigma^{1}}-\int_{\sigma^{1}}^{t \wedge \sigma^{2}} e^{-A\left(t \wedge \sigma^{2}-s\right)} B(y(s)) \mathrm{d} s \\
& +\int_{\sigma^{1}}^{t \wedge \sigma^{2}} e^{-A\left(t \wedge \sigma^{2}-s\right)} g(y(s)) \mathrm{d} s+\int_{\sigma^{1}}^{t \wedge \sigma^{2}} e^{-A\left(t \wedge \sigma^{2}-s\right)} G(y(s)) \mathrm{d} w(s) .
\end{aligned}
$$

Combining the above two relations, and using Proposition 7.1, we obtain

$$
\begin{aligned}
y\left(t \wedge \sigma^{2}\right)= & e^{-A t \wedge \sigma^{2}} y_{0}-\int_{0}^{t \wedge \sigma^{2}} e^{-A\left(t \wedge \sigma^{2}-s\right)} B(y(s)) \mathrm{d} s \\
& +\int_{0}^{t \wedge \sigma^{2}} e^{-A\left(t \wedge \sigma^{2}-s\right)} g(y(s)) \mathrm{d} s+\int_{0}^{t \wedge \sigma^{2}} e^{-A\left(t \wedge \sigma^{2}-s\right)} G(y(s)) \mathrm{d} w(s) .
\end{aligned}
$$

This can be carried over to obtain (6.1).

Let us show the uniqueness. Indeed, consider two solutions $y(t), y^{*}(t)$, and let $\sigma^{k}, \sigma^{*, k}$, be the corresponding exit times

$$
\begin{aligned}
\sigma^{k} & =\inf \left\{0 \leq t \leq T|| A^{1-\rho} y(t) \mid \geq N_{0}+k\right\} \\
\sigma^{*, k} & =\inf \left\{0 \leq t \leq T|| A^{1-\rho} y^{*}(t) \mid \geq N_{0}+k\right\} .
\end{aligned}
$$


We can write

$$
\begin{aligned}
& y\left(t \wedge \sigma^{k} \wedge \sigma^{*, k}\right)-y^{*}\left(t \wedge \sigma^{k} \wedge \sigma^{*, k}\right)=-\int_{0}^{t \wedge \sigma^{k} \wedge \sigma^{*, k}} e^{-A\left(t \wedge \sigma^{k} \wedge \sigma^{*, k}-s\right)}\left(B_{N_{0}+k}(y(s))-B_{N_{0}+k}\left(y^{*}(s)\right)\right) \mathrm{d} s \\
& +\int_{0}^{t \wedge \sigma^{k} \wedge \sigma^{*, k}} e^{-A\left(t \wedge \sigma^{k} \wedge \sigma^{*, k}-s\right)}\left(g(y(s))-g\left(y^{*}(s)\right)\right) \mathrm{d} s+\int_{0}^{t \wedge \sigma^{k} \wedge \sigma^{*, k}} e^{-A\left(t \wedge \sigma^{k} \wedge \sigma^{*, k}-s\right)}\left(G(y(s))-G\left(y^{*}(s)\right)\right) \mathrm{d} w(s) .
\end{aligned}
$$

For $t \leq T_{1}$, sufficiently small, we can proceed with a contraction argument, as in Theorem 8.1 of Appendix 2 . This will prove that

$$
y\left(t \wedge \sigma^{k} \wedge \sigma^{*, k}\right)=y^{*}\left(t \wedge \sigma^{k} \wedge \sigma^{*, k}\right), \quad t \in\left[0, T_{1}\right] .
$$

The argument can be reproduced on $\left[T_{1}, 2 T_{1}\right]$, and so on . So we obtain

$$
y\left(t \wedge \sigma^{k} \wedge \sigma^{*, k}\right)=y^{*}\left(t \wedge \sigma^{k} \wedge \sigma^{*, k}\right), \quad t \in[0, T] .
$$

Necessarily

$$
\sigma^{k}=\sigma^{*, k}
$$

and

$$
\tilde{\sigma}=\tilde{\sigma}^{*}
$$

From

$$
y\left(t \wedge \sigma^{k}\right)=y^{*}\left(t \wedge \sigma^{k}\right)
$$

and $t<\tilde{\sigma}$, we can let $k \rightarrow \infty$, and obtain, from the continuity of trajectories

$$
y(t)=y^{*}(t), \text { a.s. }
$$

Finally, assume $\tilde{\sigma}<T$, then $\sigma^{k}<T$, which implies

$$
\left|A^{1-\rho} y\left(\sigma^{k}\right)\right|=N_{0}+k .
$$

Therefore

$$
\left|A^{1-\rho} y(t)\right| \uparrow \infty, \text { as } t \uparrow \tilde{\sigma} .
$$

The proof has been completed.

\section{Appendix 1}

We are concerned here with the study of the stochastic convolution

$$
Y(t)=\int_{0}^{t} e^{-A(t-s)} \Phi(s) \mathrm{d} w(s)
$$

where $\Phi(t)$ is an adapted process such that $A^{-\beta} \Phi(t)$ belongs to $H$ for $0 \leq \beta<\frac{1}{2}$, so with values in $\left(D\left(A^{\beta}\right)\right)^{*}$. We shall make the following assumption

$$
E \sup _{0 \leq s \leq T}\left|A^{-\beta} \Phi(s)\right|^{2 r}<+\infty, \quad \beta+\frac{1}{2 r}<\frac{1}{2}, \beta \geq 0, r>0
$$

so in particular

$$
r>1 .
$$


We follow here Da Prato, Zabczyk [3], with some complements. One of the difficulties in studying the process $Y(t)$ is the dependence in $t$ inside the integral. For any fixed $t$, it is an ordinary stochastic integral, but moving $t$ forbids to apply the standard properties of stochastic integrals. For example $Y(t)$ is not a martingale. We begin by checking the following estimate

Theorem 7.1. With the assumption (7.1), then one has

$$
E|Y(t)-Y(s)|^{2 r} \leq C_{r}\left(E \sup _{0 \leq \sigma \leq t}\left|A^{-\beta} \Phi(\sigma)\right|^{2 r}\right)\left(\frac{(t-s)^{\varepsilon}}{\varepsilon}\right)^{2 r} \frac{t^{r(1-2(\varepsilon+\beta))}}{(1-2(\varepsilon+\beta))^{r}}, 0 \leq s \leq t
$$

for any $\varepsilon$ such that

$$
\frac{1}{2 r}<\varepsilon<\frac{1}{2}-\beta
$$

and the constant $C_{r}$ depends only on $r$. The process $Y(t)$ has a continuous modification which is Hölder with any exponent strictly smaller than $\frac{1}{2}-\beta-\frac{1}{2 r}$. Moreover, for any $\varepsilon$ as above, one has also

$$
\left(E \sup _{0 \leq t \leq T}|Y(t)|^{2 r}\right)^{\frac{1}{2 r}} \leq C_{r}\left(E \sup _{0 \leq \sigma \leq T}\left|A^{-\beta} \Phi(\sigma)\right|^{2 r}\right)^{\frac{1}{2 r}} \frac{T^{\frac{1}{2}-\beta} \frac{\sin \pi \varepsilon}{\pi}}{(1-2(\varepsilon+\beta))^{\frac{1}{2}}(2 \varepsilon r-1)^{1-\frac{1}{2 r}}} .
$$

Proof.

Proof of estimate (7.3):

Take $0 \leq s \leq t$. We can write

$$
Y(t)-Y(s)=\int_{s}^{t} e^{-A(t-\sigma)} \Phi(\sigma) \mathrm{d} w(\sigma)+\left(e^{-A(t-s)}-I\right) Y(s) .
$$

By the norm inequality

$$
\left(E|Y(t)-Y(s)|^{2 r}\right)^{\frac{1}{2 r}} \leq\left(E\left|\int_{s}^{t} e^{-A(t-\sigma)} \Phi(\sigma) \mathrm{d} w(\sigma)\right|^{2 r}\right)^{\frac{1}{2 r}}+\left(E\left|\left(e^{-A(t-s)}-I\right) Y(s)\right|^{2 r}\right)^{\frac{1}{2 r}}
$$

and by the Burkholder-Davis-Gundy inequality, see I. Karatzas, Shreve [6] one has

$$
\begin{gathered}
\leq C_{r}\left(E\left(\int_{s}^{t}\left|e^{-A(t-\sigma)} \Phi(\sigma)\right|^{2} \mathrm{~d} \sigma\right)^{r}\right)^{\frac{1}{2 r}}+ \\
+C_{r}\left(E\left(\int_{0}^{s}\left|\left(e^{-A(t-s)}-I\right) e^{-A(s-\sigma)} \Phi(\sigma)\right|^{2} \mathrm{~d} \sigma\right)^{r}\right)^{\frac{1}{2 r}} \\
=I_{1}+I_{2} .
\end{gathered}
$$

In $I_{1}$, we write

$$
e^{-A(t-\sigma)} \Phi(\sigma)=A^{\beta} e^{-A(t-\sigma)} A^{-\beta} \Phi(\sigma)
$$

and thus, by using the estimates on the semi group,

$$
\begin{aligned}
I_{1} & \leq C_{r}\left(E\left(\int_{s}^{t} \frac{\left|A^{-\beta} \Phi(\sigma)\right|^{2}}{(t-\sigma)^{2 \beta}} \mathrm{d} \sigma\right)^{r}\right)^{\frac{1}{2 r}} \\
& \leq C_{r}\left(E \sup _{s \leq \sigma \leq t}\left|A^{-\beta} \Phi(\sigma)\right|^{2 r}\right)^{\frac{1}{2 r}} \frac{(t-s)^{\frac{1}{2}-\beta}}{(1-2 \beta)^{\frac{1}{2}}}
\end{aligned}
$$


which we may also majorize by

$$
I_{1} \leq C_{r}\left(E \sup _{0 \leq \sigma \leq t}\left|A^{-\beta} \Phi(\sigma)\right|^{2 r}\right)^{\frac{1}{2 r}} \frac{(t-s)^{\varepsilon} t^{\frac{1}{2}-(\varepsilon+\beta)}}{(1-2 \beta)^{\frac{1}{2}}}
$$

with any $\varepsilon$ satisfying (7.4).

We turn now to $I_{2}$. We first write

$$
\begin{aligned}
\left(e^{-A(t-s)}-I\right) e^{-A(s-\sigma)} \Phi(\sigma) & =(t-s) \int_{0}^{1} \mathrm{~d} \theta e^{-A \theta(t-s)} A e^{-A(s-\sigma)} \Phi(\sigma) \\
& =(t-s)^{\varepsilon} \int_{0}^{1} \mathrm{~d} \theta(t-s)^{1-\varepsilon} e^{-A \theta(t-s)} A^{1-\varepsilon} A^{\varepsilon} e^{-A(s-\sigma)} \Phi(\sigma)
\end{aligned}
$$

and thus

$$
\left|\left(e^{-A(t-s)}-I\right) e^{-A(s-\sigma)} \Phi(\sigma)\right| \leq c \frac{(t-s)^{\varepsilon}}{\varepsilon}\left|A^{\varepsilon} e^{-A(s-\sigma)} \Phi(\sigma)\right| .
$$

Thus

$$
I_{2} \leq C_{r} \frac{(t-s)^{\varepsilon}}{\varepsilon}\left(E\left(\int_{0}^{s}\left|A^{\varepsilon} e^{-A(s-\sigma)} \Phi(\sigma)\right|^{2} \mathrm{~d} \sigma\right)^{r}\right)^{\frac{1}{2 r}} .
$$

Introducing the term $A^{-\beta}$ as above, we obtain

$$
I_{2} \leq C_{r} \frac{(t-s)^{\varepsilon}}{\varepsilon}\left(E\left(\int_{0}^{s} \frac{\left|A^{-\beta} \Phi(\sigma)\right|^{2}}{(s-\sigma)^{2(\varepsilon+\beta)}} \mathrm{d} \sigma\right)^{r}\right)^{\frac{1}{2 r}}
$$

and finally

$$
I_{2} \leq C_{r}\left(E \sup _{0 \leq \sigma \leq t}\left|A^{-\beta} \Phi(\sigma)\right|^{2 r}\right)^{\frac{1}{2 r}} \frac{(t-s)^{\varepsilon}}{\varepsilon} \frac{t^{\frac{1}{2}-(\varepsilon+\beta)}}{(1-2(\varepsilon+\beta))^{\frac{1}{2}}} .
$$

Adjusting the constants we get

$$
I_{1}+I_{2} \leq C_{r}\left(E \sup _{0 \leq \sigma \leq t}\left|A^{-\beta} \Phi(\sigma)\right|^{2 r}\right)^{\frac{1}{2 r}} \frac{(t-s)^{\varepsilon}}{\varepsilon} \frac{t^{\frac{1}{2}-(\varepsilon+\beta)}}{(1-2(\varepsilon+\beta))^{\frac{1}{2}}} .
$$

The estimate (7.3) follows immediately.

We then use the Kolmogorov-Čentsov Theorem, see [6], to conclude that $Y(t)$ has a continuous version which is Hölder with exponent strictly smaller than $\varepsilon-\frac{1}{2 r}$, and therefore any exponent strictly smaller than $\frac{1}{2}-\beta-\frac{1}{2 r}$ is fine.

We turn now to estimate (7.5). Again the difficulty is that we cannot use the usual martingale estimates. One way to proceed, following Da Prato, Zabczyk [3] is to make use of the identity

$$
\frac{\pi}{\sin \pi \varepsilon}=\int_{\sigma}^{t}(t-s)^{\varepsilon-1}(s-\sigma)^{-\varepsilon} \mathrm{d} s, \forall \sigma \leq t, 0<\varepsilon<1 .
$$

Then we can write obviously

$$
Y(t)=\frac{\sin \pi \varepsilon}{\pi} \int_{0}^{t} e^{-A(t-\sigma)}\left[\int_{\sigma}^{t}(t-s)^{\varepsilon-1}(s-\sigma)^{-\varepsilon} \mathrm{d} s\right] \Phi(\sigma) \mathrm{d} w(\sigma)
$$


and from Fubini's Theorem we derive the formula

$$
Y(t)=\frac{\sin \pi \varepsilon}{\pi} \int_{0}^{t}(t-s)^{\varepsilon-1} e^{-A(t-s)} \Psi(s) \mathrm{d} s
$$

with

$$
\Psi(s)=\int_{0}^{s}(s-\sigma)^{-\varepsilon} e^{-A(s-\sigma)} \Phi(\sigma) \mathrm{d} w(\sigma)
$$

We can then derive from (7.7), making use of Hölder's inequality

$$
\left(E \sup _{0 \leq t \leq T}|Y(t)|^{2 r}\right)^{\frac{1}{2 r}} \leq C_{r} \frac{\sin \pi \varepsilon}{\pi}\left(E \int_{0}^{T}|\Psi(t)|^{2 r} \mathrm{~d} t\right)^{\frac{1}{2 r}} \frac{T^{\varepsilon-\frac{1}{2 r}}}{(2 r \varepsilon-1)^{1-\frac{1}{2 r}}}
$$

recalling the condition $2 r \varepsilon>1$. Next, we have

$$
\begin{aligned}
E|\Psi(t)|^{2 r} & =E\left|\int_{0}^{t}(t-\sigma)^{-\varepsilon} e^{-A(t-\sigma)} \Phi(\sigma) \mathrm{d} w(\sigma)\right|^{2 r} \\
& \leq C_{r} E\left(\int_{0}^{t}(t-\sigma)^{-2 \varepsilon}\left|e^{-A(t-\sigma)} \Phi(\sigma)\right|^{2} \mathrm{~d} \sigma\right)^{r} \\
& \leq C_{r} E\left(\int_{0}^{t} \frac{\left|A^{-\beta} \Phi(\sigma)\right|^{2}}{(t-\sigma)^{2(\varepsilon+\beta)}} \mathrm{d} \sigma\right)^{r} \\
& \leq C_{r} E \sup _{0 \leq \sigma \leq T}\left|A^{-\beta} \Phi(\sigma)\right|^{2 r} \frac{t^{r(1-2(\varepsilon+\beta))}}{(1-2(\varepsilon+\beta))^{r}}
\end{aligned}
$$

and then

$$
E \int_{0}^{T}|\Psi(t)|^{2 r} \mathrm{~d} t \leq C_{r} E \sup _{0 \leq \sigma \leq T}\left|A^{-\beta} \Phi(\sigma)\right|^{2 r} \frac{T^{r(1-2(\varepsilon+\beta))+1}}{(1-2(\varepsilon+\beta))^{r}} .
$$

Collecting results, we obtain easily (7.5). The proof has been completed.

It is important for the following to introduce random times, with respect to the filtration $\mathcal{F}^{t}$ generated by the Wiener process in relation with the process $Y(t)$. More precisely, let $\tau, \tilde{\tau}$ be two random times such that

$$
0 \leq \tilde{\tau} \leq \tau \leq T_{0}
$$

where $T_{0}$ is deterministic. We want to consider the quantity

$$
Y_{\tilde{\tau}, \tau}=\int_{\tilde{\tau}}^{\tau} e^{-A(\tau-s)} \Phi(s) \mathrm{d} w(s)
$$

where the difficulty stems from the presence of $\tau$ inside the integral, and not just as an end point of the integration interval. Setting

$$
\Phi_{\tilde{\tau}, \tau}(s)=\mathbb{1}_{\tilde{\tau} \leq s<\tau} \Phi(s)
$$

we know that $\Phi_{\tilde{\tau}, \tau}(s)$ is still an adapted process, and we may try to mimic the usual stochastic integral by setting

$$
Y_{\tilde{\tau}, \tau}=\int_{0}^{\tau} e^{-A(\tau-s)} \Phi_{\tilde{\tau}, \tau}(s) \mathrm{d} w(s)
$$


but the process $e^{-A(\tau-s)} \Phi_{\tilde{\tau}, \tau}(s)$ is not adapted. So (7.11) cannot be defined as a stochastic integral. Of course, since the process $Y(t)$ is well defined, we can still consider

$$
\tilde{Y}(t)=\int_{0}^{t} e^{-A(t-s)} \Phi_{\tilde{\tau}, \tau}(s) \mathrm{d} w(s)
$$

which is perfectly well defined, and then set, as a definition

$$
Y_{\tilde{\tau}, \tau}(\omega)=\tilde{Y}(\tau)(\omega)
$$

But, this definition is not sufficiently convenient, as it cannot be expressed as a stochastic integral. In this context, formulas $(7.7),(7.8)$ are very useful. In particular, we have

$$
Y_{\tilde{\tau}, \tau}=\frac{\sin \pi \varepsilon}{\pi} \int_{0}^{\tau}(\tau-s)^{\varepsilon-1} e^{-A(\tau-s)} \Psi_{\tilde{\tau}, \tau}(s) \mathrm{d} s
$$

with

$$
\Psi_{\tilde{\tau}, \tau}(s)=\int_{0}^{s}(s-\sigma)^{-\varepsilon} e^{-A(s-\sigma)} \Phi_{\tilde{\tau}, \tau}(\sigma) \mathrm{d} w(\sigma)
$$

and $(7.14,7.15)$ make perfect sense. Note that, among other things,

$$
\Psi_{\tilde{\tau}, \tau}(s)=\int_{s \wedge \tilde{\tau}}^{s \wedge \tau}(s-\sigma)^{-\varepsilon} e^{-A(s-\sigma)} \Phi(\sigma) \mathrm{d} w(\sigma)
$$

and the random times appear only as end points of the integration interval. The formulas $(7.14,7.15)$ will provide the convenient definition of $Y_{\tilde{\tau}, \tau}$, although we shall keep the writing of the right handside of (7.9), since it is not only mnemonic, but also compatible with the manipulations that we expect from such a writing, as we shall see now. In particular, we want to check the

Proposition 7.1. The following formula holds

$$
\int_{\tilde{\tau}}^{\tau+\tau_{0}} e^{-A\left(\tau+\tau_{0}-s\right)} \Phi(s) \mathrm{d} w(s)=e^{-A \tau_{0}} \int_{\tilde{\tau}}^{\tau} e^{-A(\tau-s)} \Phi(s) \mathrm{d} w(s)+\int_{\tau}^{\tau+\tau_{0}} e^{-A\left(\tau+\tau_{0}-s\right)} \Phi(s) \mathrm{d} w(s) .
$$

Proof. We first notice that, by using standard properties of stochastic integrals, if we define for deterministic numbers $0 \leq \tilde{t} \leq t$

$$
\Psi(\tilde{t}, t, s ; \omega)=\int_{s \wedge \tilde{t}}^{s \wedge t}(s-\sigma)^{-\varepsilon} e^{-A(s-\sigma)} \Phi(\sigma) \mathrm{d} w(\sigma)
$$

then we have a.s.

$$
\Psi_{\tilde{\tau}, \tau}(s)(\omega)=\Psi(\tilde{\tau}(\omega), \tau(\omega), s ; \omega) .
$$

We next take note of the relation, valid for any $0 \leq \tilde{t} \leq t$, and $t_{0} \geq 0$

$$
\int_{0}^{t+t_{0}}\left(t+t_{0}-s\right)^{\varepsilon-1} e^{-A\left(t+t_{0}-s\right)} \Psi(\tilde{t}, t, s ; \omega) \mathrm{d} s=e^{-A t_{0}} \int_{0}^{t}(t-s)^{\varepsilon-1} e^{-A(t-s)} \Psi(\tilde{t}, t, s ; \omega) \mathrm{d} s .
$$


Indeed, expressing the left hand side of (7.20) with (7.18), we have

$$
\begin{aligned}
& \int_{0}^{t+t_{0}}\left(t+t_{0}-s\right)^{\varepsilon-1} e^{-A\left(t+t_{0}-s\right)} \Psi(\tilde{t}, t, s ; \omega) \mathrm{d} s= \\
& \qquad \int_{0}^{t+t_{0}}\left(t+t_{0}-s\right)^{\varepsilon-1} e^{-A\left(t+t_{0}-s\right)}\left[\int_{0}^{s}(s-\sigma)^{-\varepsilon} e^{-A(s-\sigma)} \Phi(\sigma) \mathbb{1}_{\tilde{t} \leq \sigma<t} \mathrm{~d} w(\sigma)\right] \mathrm{d} s
\end{aligned}
$$

and using Fubini

$$
\begin{aligned}
& =\int_{0}^{t+t_{0}} e^{-A\left(t+t_{0}-\sigma\right)}\left[\int_{\sigma}^{t+t_{0}}\left(t+t_{0}-s\right)^{\varepsilon-1}(s-\sigma)^{-\varepsilon} \mathrm{d} s\right] \Phi(\sigma) \mathbb{1}_{\tilde{t} \leq \sigma<t} \mathrm{~d} w(\sigma) \\
& =e^{-A t_{0}} \int_{0}^{t} e^{-A(t-\sigma)}\left[\int_{\sigma}^{t}(t-s)^{\varepsilon-1}(s-\sigma)^{-\varepsilon} \mathrm{d} s\right] \Phi(\sigma) \mathbb{1}_{\tilde{t} \leq \sigma<t} \mathrm{~d} w(\sigma)
\end{aligned}
$$

where we have used the fact that the integrand vanishes, for $\sigma \geq t$, and of the identity (7.6). So (7.20) is obtained.

In replacing, for any $\omega, \tilde{t}$ by $\tilde{\tau}, t$ by $\tau$, and $t_{0}$ by $\tau_{0}$, where $\tau_{0}$ is another random time, and recalling (7.19), we can write

$$
\int_{0}^{\tau+\tau_{0}}\left(\tau+\tau_{0}-s\right)^{\varepsilon-1} e^{-A\left(\tau+\tau_{0}-s\right)} \Psi_{\tilde{\tau}, \tau}(s) \mathrm{d} s=e^{-A \tau_{0}} \int_{0}^{\tau}(\tau-s)^{\varepsilon-1} e^{-A(\tau-s)} \Psi_{\tilde{\tau}, \tau}(s) \mathrm{d} s .
$$

Now, to check (7.17) means

$$
Y_{\tilde{\tau}, \tau+\tau_{0}}=e^{-A \tau_{0}} Y_{\tilde{\tau}, \tau}+Y_{\tau, \tau+\tau_{0}}
$$

or from (7.14)

$$
\begin{aligned}
\int_{0}^{\tau+\tau_{0}}\left(\tau+\tau_{0}-s\right)^{\varepsilon-1} e^{-A\left(\tau+\tau_{0}-s\right)} \Psi_{\tilde{\tau}, \tau+\tau_{0}}(s) \mathrm{d} s= & e^{-A \tau_{0}} \int_{0}^{\tau}(\tau-s)^{\varepsilon-1} e^{-A(\tau-s)} \Psi_{\tilde{\tau}, \tau}(s) \mathrm{d} s \\
& +\int_{0}^{\tau+\tau_{0}}\left(\tau+\tau_{0}-s\right)^{\varepsilon-1} e^{-A\left(\tau+\tau_{0}-s\right)} \Psi_{\tau, \tau+\tau_{0}}(s) \mathrm{d} s
\end{aligned}
$$

But it is easy to check that

$$
\Psi_{\tilde{\tau}, \tau+\tau_{0}}-\Psi_{\tau, \tau+\tau_{0}}=\Psi_{\tilde{\tau}, \tau}
$$

and thus (7.23) amounts to (7.21) and thus is proven. The proof has been completed.

We can state also the somewhat natural

Proposition 7.2. The variable $Y_{\tilde{\tau}, \tau}$ is $\mathcal{F}^{\tau}$ measurable

Proof.

Let $B$ be any Borel set of $H$. We must check that

$$
\left\{Y_{\tilde{\tau}, \tau} \in B\right\} \cap\{\tau \leq t\} \subset \mathcal{F}^{t}
$$

However considering formula (7.14), if $\tau \leq t$, we can also write

$$
Y_{\tilde{\tau}, \tau}=\frac{\sin \pi \varepsilon}{\pi} \int_{0}^{t}(\tau-s)^{\varepsilon-1} e^{-A(\tau-s)} \Psi_{\tilde{\tau}, \tau}(s) \Pi_{s<\tau} \mathrm{d} s
$$


and the integrand is, for each $s, \mathcal{F}^{s}$ measurable, hence also $\mathcal{F}^{t}$ measurable. Therefore the integral on the right handside is $\mathcal{F}^{t}$ measurable. Hence (7.24) is obtained. The proof has been completed.

Now from (7.13), we can write

$$
E\left|Y_{\tilde{\tau}, \tau}\right|^{2 r} \leq E \sup _{0 \leq t \leq T_{0}}|\tilde{Y}(t)|^{2 r}
$$

and making use of (7.5), we obtain

$$
\left(E\left|Y_{\tilde{\tau}, \tau}\right|^{2 r}\right)^{\frac{1}{2 r}} \leq C_{r}\left(E \sup _{\tilde{\tau} \leq \sigma \leq \tau}\left|A^{-\beta} \Phi(\sigma)\right|^{2 r}\right)^{\frac{1}{2 r}} \frac{T_{0}^{\frac{1}{2}-\beta}}{(1-2(\varepsilon+\beta))^{\frac{1}{2}}(2 \varepsilon r-1)^{1-\frac{1}{2 r}}} \frac{\sin \pi \varepsilon}{\pi} .
$$

Consider finally the process

$$
Y(t \wedge \tau)=\int_{0}^{t \wedge \tau} e^{-A(t \wedge \tau-s)} \Phi(s) \mathrm{d} w(s)
$$

where $\tau$ is a random time. Then we have the

Proposition 7.3. The process $Y(t \wedge \tau)$ has a continuous modification which is Hölder with exponent strictly smaller than $\frac{1}{2}-\beta-\frac{1}{r}$.

Proof. Let $0<s<t$, we know from Proposition 7.1 that

$$
Y(t \wedge \tau)-Y(s \wedge \tau)=\int_{s \wedge \tau}^{t \wedge \tau} e^{-A(t \wedge \tau-\sigma)} \Phi(\sigma) \mathrm{d} w(\sigma)+\left(e^{-A(t \wedge \tau-s \wedge \tau)}-I\right) Y(s \wedge \tau) .
$$

By the norm inequality, we can write

$$
\begin{aligned}
\left(E|Y(t \wedge \tau)-Y(s \wedge \tau)|^{2 r}\right)^{\frac{1}{2 r}} \leq & \left(E\left|\int_{s \wedge \tau}^{t \wedge \tau} e^{-A(t \wedge \tau-\sigma)} \Phi(\sigma) \mathrm{d} w(\sigma)\right|^{2 r}\right)^{\frac{1}{2 r}} \\
& +\left(E\left|\left(e^{-A(t \wedge \tau-s \wedge \tau)}-I\right) Y(s \wedge \tau)\right|^{2 r}\right)^{\frac{1}{2 r}}=I_{1}+I_{2} .
\end{aligned}
$$

We can write, using (7.6)

$$
\int_{s \wedge \tau}^{t \wedge \tau} e^{-A(t \wedge \tau-\sigma)} \Phi(\sigma) \mathrm{d} w(\sigma)=\frac{\sin \pi \varepsilon}{\pi} \int_{s \wedge \tau}^{t \wedge \tau}(t \wedge \tau-\theta)^{\varepsilon-1} e^{-A(t \wedge \tau-\theta)} \Psi_{s \wedge \tau}(\theta) \mathrm{d} \theta
$$

with

From Hölder's inequality, we derive

$$
\Psi_{s \wedge \tau}(\theta)=\int_{0}^{\theta}(\theta-\sigma)^{-\varepsilon} \Pi_{s \wedge \tau \leq \sigma} e^{-A(\theta-\sigma)} \Phi(\sigma) \mathrm{d} w(\sigma) .
$$

$$
I_{1} \leq C_{r} \frac{\sin \pi \varepsilon}{\pi} \frac{(t-s)^{\varepsilon-\frac{1}{2 r}}}{(2 r \varepsilon-1)^{1-\frac{1}{2 r}}}\left(E \int_{s}^{t}\left|\Psi_{s \wedge \tau}(\theta)\right|^{2 r} \mathrm{~d} \theta\right)^{\frac{1}{2 r}}
$$

Next

$$
\begin{aligned}
E\left|\Psi_{s \wedge \tau}(\theta)\right|^{2 r} & \leq C_{r} E\left(\int_{0}^{\theta}(\theta-\sigma)^{-2 \varepsilon}\left|e^{-A(\theta-\sigma)} \Phi(\sigma)\right|^{2} d \sigma\right)^{r} \\
& \leq C_{r} E\left(\int_{0}^{\theta} \frac{\left|A^{-\beta} \Phi(\sigma)\right|^{2}}{(\theta-\sigma)^{2(\varepsilon+\beta)}} \mathrm{d} \sigma\right)^{r}
\end{aligned}
$$


and then

$$
\leq C_{r} E \sup _{0 \leq \sigma \leq t}\left|A^{-\beta} \Phi(\sigma)\right|^{2 r} \frac{\theta^{r(1-2(\varepsilon+\beta))}}{(1-2(\varepsilon+\beta))^{r}}
$$

Collecting results, we get

$$
E \int_{s}^{t}\left|\Psi_{s \wedge \tau}(\theta)\right|^{2 r} \mathrm{~d} \theta \leq C_{r} E \sup _{0 \leq \sigma \leq t}\left|A^{-\beta} \Phi(\sigma)\right|^{2 r}(t-s) \frac{t^{r(1-2(\varepsilon+\beta))}}{(1-2(\varepsilon+\beta))^{r}}
$$

$$
I_{1} \leq C_{r} \frac{\sin \pi \varepsilon}{\pi} \frac{(t-s)^{\varepsilon}}{(2 r \varepsilon-1)^{1-\frac{1}{2 r}}}\left(E \sup _{0 \leq \sigma \leq t}\left|A^{-\beta} \Phi(\sigma)\right|^{2 r}\right)^{\frac{1}{2 r}} \frac{t^{\left(\frac{1}{2}-(\varepsilon+\beta)\right)}}{(1-2(\varepsilon+\beta))^{\frac{1}{2}}} .
$$

We turn to $I_{2}$, which we express using

$$
\left(e^{-A(t \wedge \tau-s \wedge \tau)}-I\right) Y(s \wedge \tau)=\frac{\sin \pi \varepsilon}{\pi} \int_{0}^{s \wedge \tau}(s \wedge \tau-\theta)^{\varepsilon-1}\left(e^{-A(t \wedge \tau-s \wedge \tau)}-I\right) e^{-A(s \wedge \tau-\theta)} \Psi(\theta) \mathrm{d} \theta
$$

with

$$
\Psi(\theta)=\int_{0}^{\theta}(\theta-\sigma)^{-\varepsilon} e^{-A(\theta-\sigma)} \Phi(\sigma) \mathrm{d} w(\sigma)
$$

Then

$$
\left|\left(e^{-A(t \wedge \tau-s \wedge \tau)}-I\right) Y(s \wedge \tau)\right| \leq \frac{\sin \pi \varepsilon}{\pi} \frac{(t-s)^{\varepsilon^{\prime}}}{\varepsilon^{\prime}} \int_{0}^{s \wedge \tau}(s \wedge \tau-\theta)^{\varepsilon-\varepsilon^{\prime}-1}|\Psi(\theta)| d \theta .
$$

Using Hölder's inequality we obtain

$$
\left|\left(e^{-A(t \wedge \tau-s \wedge \tau)}-I\right) Y(s \wedge \tau)\right| \leq \frac{\sin \pi \varepsilon}{\pi} \frac{(t-s)^{\varepsilon^{\prime}}}{\varepsilon^{\prime}}\left(\int_{0}^{s}|\Psi(\theta)|^{2 r} \mathrm{~d} \theta\right)^{\frac{1}{2 r}} \frac{s^{\varepsilon-\varepsilon^{\prime}-\frac{1}{2 r}}}{\left(2 r\left(\varepsilon-\varepsilon^{\prime}\right)-1\right)^{1-\frac{1}{2 r}}} .
$$

It follows that

$$
I_{2} \leq \frac{\sin \pi \varepsilon}{\pi} \frac{(t-s)^{\varepsilon^{\prime}}}{\varepsilon^{\prime}}\left(E \int_{0}^{s}|\Psi(\theta)|^{2 r} \mathrm{~d} \theta\right)^{\frac{1}{2 r}} \frac{s^{\varepsilon-\varepsilon^{\prime}-\frac{1}{2 r}}}{\left(2 r\left(\varepsilon-\varepsilon^{\prime}\right)-1\right)^{1-\frac{1}{2 r}}}
$$

Also

$$
E|\Psi(\theta)|^{2 r} \leq C_{r} E\left(\int_{0}^{\theta} \frac{\left|A^{-\beta} \Phi(\sigma)\right|^{2}}{(\theta-\sigma)^{2(\varepsilon+\beta)}} \mathrm{d} \sigma\right)^{r}
$$

which, combined with the previous estimate, yields

$$
I_{2} \leq C_{r} \frac{\sin \pi \varepsilon}{\pi} \frac{(t-s)^{\varepsilon^{\prime}}}{\varepsilon^{\prime}}\left(E \sup _{0 \leq \sigma \leq s}\left|A^{-\beta} \Phi(\sigma)\right|^{2 r}\right)^{\frac{1}{2 r}} \frac{s^{\frac{1}{2}-\beta-\varepsilon^{\prime}}}{\left(2 r\left(\varepsilon-\varepsilon^{\prime}\right)-1\right)^{1-\frac{1}{2 r}}(1-2(\varepsilon+\beta))^{\frac{1}{2}}} .
$$

Adjusting the estimates for $I_{1}$ and $I_{2}$, we obtain easily

$$
E|Y(t \wedge \tau)-Y(s \wedge \tau)|^{2 r} \leq C_{r}\left(\frac{\sin \pi \varepsilon}{\pi}\right)^{2 r} \frac{(t-s)^{2 r \varepsilon^{\prime}}}{{\varepsilon^{\prime 2}}^{2 r}}\left(E \sup _{0 \leq \sigma \leq t}\left|A^{-\beta} \Phi(\sigma)\right|^{2 r}\right) \frac{t^{r\left(1-2 \beta-2 \varepsilon^{\prime}\right.}}{\left(2 r\left(\varepsilon-\varepsilon^{\prime}\right)-1\right)^{2 r-1}(1-2(\varepsilon+\beta))^{r}}
$$


Let then pick

and choose $\varepsilon, \varepsilon^{\prime}$ such that

$$
\delta<\frac{1}{2}-\beta-\frac{1}{r}
$$

$$
\frac{1}{2}-\beta>\varepsilon>\varepsilon^{\prime}+\frac{1}{2 r}>\delta+\frac{1}{r}
$$

then from the Kolmogorov-ČCentsov Theorem again, we obtain the desired property. The proof has been completed.

\section{Appendix 2}

\subsection{Statement of the problem and results}

We consider a Lipschitz bounded approximation of the vorticity operator $B$, as follows

$$
B_{M}(u)=B(u)\left[\mathbb{I}_{\left\{\left|A^{1-\rho} u\right| \leq M\right\}}+\left(M+1-\left|A^{1-\rho} u\right|\right) \amalg_{\left\{M<\left|A^{1-\rho} u\right| \leq M+1\right\}}\right]
$$

then as easily seen

$$
\begin{aligned}
\left|B_{M}(u)\right| & \leq c(M+1)^{2} \\
\left|B_{M}(u)-B_{M}(v)\right| & \leq c(M+1)^{2}\left|A^{1-\rho}(u-v)\right| .
\end{aligned}
$$

We next consider functions $g(u), G(u)$ defined also on $D\left(A^{1-\rho} u\right)$, satisfying

$$
\begin{array}{ll}
\left|A^{-\rho^{\prime}}(g(u)-g(v))\right| \leq K\left|A^{1-\rho}(u-v)\right|, & 0 \leq \rho^{\prime}<\rho \\
\left|A^{\varepsilon^{\prime}}(G(u)-G(v))\right| \leq K\left|A^{1-\rho}(u-v)\right|, \quad 1 \geq \varepsilon^{\prime}+\rho>\frac{1}{2} .
\end{array}
$$

Let us consider a probability system $\Omega, \mathcal{A}, P$, with an $m$ dimensional Wiener process, and let

$$
\mathcal{F}^{t}=\sigma-\text { algebra generated by } w(s), 0 \leq s \leq t .
$$

Let $\tau$ be a random time, with

$$
\tau \leq T_{0} \text { deterministic. }
$$

We consider a random variable

$$
y_{\tau} \text { is } \mathcal{F}^{\tau} \text { measurable, }\left(E\left|A^{1-\rho} y_{\tau}\right|^{2 r}\right)^{\frac{1}{2 r}}<\infty
$$

with

$$
\varepsilon^{\prime}+\rho>\frac{1}{2}+\frac{1}{2 r}
$$

We are interested in the following equation

$$
\begin{aligned}
y(t)= & E\left[e^{-A(t-\tau)^{+}} y_{\tau} \mid \mathcal{F}^{t}\right]-\int_{0}^{t} \mathbb{I}_{s \geq \tau} e^{-A(t-s)} B_{M}(y(s)) \mathrm{d} s \\
& +\int_{0}^{t} \mathbb{I}_{s \geq \tau} e^{-A(t-s)} g(y(s)) \mathrm{d} s+\int_{0}^{t} \mathbb{I}_{s \geq \tau} e^{-A(t-s)} G(y(s)) \mathrm{d} w(s)
\end{aligned}
$$


We want to prove the following:

Theorem 8.1. We make the assumptions (8.1, 8.4, 8.5, 8.6, 8.7, 8.8), then there exists a unique solution of (8.9), such that

$$
\left(E \sup _{0 \leq t \leq T_{0}}\left|A^{1-\rho} y(t)\right|^{2 r}\right)^{\frac{1}{2 r}} \leq C\left[(1+M)^{2}+\left(E\left|A^{1-\rho} y_{\tau}\right|^{2 r}\right)^{\frac{1}{2 r}}\right]
$$

and

$$
y(t) \text { is an adapted process, } y(t)-E\left[e^{-A(t-\tau)^{+}} y_{\tau} \mid \mathcal{F}^{t}\right]
$$

has a continuous modification with values in $D\left(A^{1-\rho}\right)$, which is Hölder with exponent strictly smaller than $\min \left(\rho-\rho^{\prime}, \varepsilon^{\prime}+\rho-\frac{1}{2}-\frac{1}{2 r}\right)$.

Proof. Let for $T \leq T_{0}, \mathcal{H}_{T}$ be the Banach space of processes $z$ such that

$$
\|\mid\| z \|_{T}=\left(E \sup _{0 \leq t \leq T}\left|A^{1-\rho} z(t)\right|^{2 r}\right)^{\frac{1}{2 r}}<\infty .
$$

Consider for $\zeta \in \mathcal{H}_{T}$, the map

$$
\begin{aligned}
z= & \mathcal{T}(\zeta)=E\left[e^{-A(t-\tau)^{+}} y_{\tau} \mid \mathcal{F}^{t}\right]-\int_{0}^{t} \mathbb{I}_{s \geq \tau} e^{-A(t-s)} B_{M}(\zeta(s)) \mathrm{d} s \\
& +\int_{0}^{t} \mathbb{I}_{s \geq \tau} e^{-A(t-s)} g(\zeta(s)) \mathrm{d} s+\int_{0}^{t} \mathbb{I}_{s \geq \tau} e^{-A(t-s)} G(\zeta(s)) \mathrm{d} w(s) .
\end{aligned}
$$

We first check that $z \in \mathcal{H}_{T}$. Indeed, from martingale properties

$$
\begin{aligned}
E \sup _{0 \leq t \leq T}\left|A^{1-\rho} E\left[e^{-A(t-\tau)^{+}} y_{\tau} \mid \mathcal{F}^{t}\right]\right|^{2 r} & \leq C_{r} E\left|E\left[A^{1-\rho} e^{-A(T-\tau)^{+}} y_{\tau} \mid \mathcal{F}^{T}\right]\right|^{2 r} \\
& \leq C_{r} E\left|A^{1-\rho} e^{-A(T-\tau)^{+}} y_{\tau}\right|^{2 r}
\end{aligned}
$$

Therefore

$$
\left(E \sup _{0 \leq t \leq T}\left|A^{1-\rho} E\left[e^{-A(t-\tau)^{+}} y_{\tau} \mid \mathcal{F}^{t}\right]\right|^{2 r}\right)^{\frac{1}{2 r}} \leq C_{r}\left(E\left|A^{1-\rho} y_{\tau}\right|^{2 r}\right)^{\frac{1}{2 r}}
$$

We note that

$$
\sup _{0 \leq t \leq T}\left|A^{1-\rho} \int_{0}^{t} \mathbb{I}_{s \geq \tau} e^{-A(t-s)} B_{M}(\zeta(s)) \mathrm{d} s\right| \leq c(M+1)^{2} \frac{T^{\rho}}{\rho} .
$$

We next have

$$
\begin{gathered}
E \sup _{0 \leq t \leq T}\left|\int_{0}^{t} \mathbb{I}_{s \geq \tau} A^{1-\rho} e^{-A(t-s)} g(\zeta(s)) \mathrm{d} s\right|^{2 r}=E \sup _{0 \leq t \leq T}\left|\int_{0}^{t} \mathbb{I}_{s \geq \tau} A^{1-\rho+\rho^{\prime}} e^{-A(t-s)} A^{-\rho^{\prime}} g(\zeta(s)) \mathrm{d} s\right|^{2 r} \\
\leq K E \sup _{0 \leq t \leq T}\left(\int_{0}^{t} \frac{1+\left|A^{1-\rho} \zeta(s)\right|}{(t-s)^{1-\rho+\rho^{\prime}}} \mathrm{d} s\right)^{2 r} \\
\leq K E\left[\frac{T^{\rho-\rho^{\prime}}}{\rho-\rho^{\prime}}\left(1+\sup _{0 \leq t \leq T}\left|A^{1-\rho} \zeta(t)\right|\right)\right]^{2 r}
\end{gathered}
$$


Therefore, from the norm inequality, we get

$$
\left(E \sup _{0 \leq t \leq T}\left|\int_{0}^{t} \mathbb{I}_{s \geq \tau} A^{1-\rho} e^{-A(t-s)} g(\zeta(s)) \mathrm{d} s\right|^{2 r}\right)^{\frac{1}{2 r}} \leq K \frac{T^{\rho-\rho^{\prime}}}{\rho-\rho^{\prime}}\left(1+\left(E \sup _{0 \leq t \leq T}\left|A^{1-\rho} \zeta(t)\right|^{2 r}\right)^{\frac{1}{2 r}}\right) .
$$

Finally, setting

$$
Y(t)=\int_{0}^{t} \mathbb{I}_{s \geq \tau} A^{1-\rho} e^{-A(t-s)} G(\zeta(s)) \mathrm{d} w(s)
$$

and

$$
\Phi(s)=\mathbb{1}_{s \geq \tau} A^{1-\rho} G(\zeta(s))
$$

we are in the situation of $(7.1,7.2)$ of Appendix 1, with

$$
\beta=1-\rho-\varepsilon^{\prime} .
$$

Hence, applying estimate (7.5), we obtain

$$
\begin{aligned}
\left(E \sup _{0 \leq t \leq T}|Y(t)|^{2 r}\right)^{\frac{1}{2 r}} & \leq C_{r, \rho, \varepsilon^{\prime}} T^{\rho+\varepsilon^{\prime}-\frac{1}{2}}\left(E \sup _{0 \leq t \leq T}\left|A^{\varepsilon^{\prime}} G(\zeta(t))\right|^{2 r}\right)^{\frac{1}{2 r}} \\
& \leq C_{r, \rho, \varepsilon^{\prime}} K T^{\rho+\varepsilon^{\prime}-\frac{1}{2}}\left(1+\left(E \sup _{0 \leq t \leq T}\left|A^{1-\rho} \zeta(t)\right|^{2 r}\right)^{\frac{1}{2 r}}\right)
\end{aligned}
$$

Hence $z \in \mathcal{H}_{T}$.

Let us show that $\mathcal{T}$ is a contraction. Indeed, pick $\zeta^{1}, \zeta^{2}$, and

$$
z^{1}=\mathcal{T}\left(\zeta^{1}\right), z^{2}=\mathcal{T}\left(\zeta^{2}\right) .
$$

Then we have

$$
\begin{aligned}
z^{1}(t)-z^{2}(t)= & -\int_{0}^{t} \mathbb{1}_{s \geq \tau} e^{-A(t-s)}\left(B_{M}\left(\zeta^{1}(s)\right)-B_{M}\left(\zeta^{2}(s)\right)\right) \mathrm{d} s \\
& +\int_{0}^{t} \mathbb{I}_{s \geq \tau} e^{-A(t-s)}\left(g\left(\zeta^{1}(s)\right)-g\left(\zeta^{2}(s)\right)\right) \mathrm{d} s+\int_{0}^{t} \mathbb{I}_{s \geq \tau} e^{-A(t-s)}\left(G\left(\zeta^{1}(s)\right)-G\left(\zeta^{2}(s)\right)\right) \mathrm{d} w(s) .
\end{aligned}
$$

Hence, it follows

$$
\begin{aligned}
\left(E \sup _{0 \leq t \leq T}\left|A^{1-\rho}\left(z^{1}(t)-z^{2}(t)\right)\right|^{2 r}\right)^{\frac{1}{2 r}} \leq & \left(E \sup _{0 \leq t \leq T}\left|\int_{0}^{t} \mathbb{I}_{s \geq \tau} A^{1-\rho} e^{-A(t-s)}\left(B_{M}\left(\zeta^{1}(s)\right)-B_{M}\left(\zeta^{2}(s)\right)\right) \mathrm{d} s\right|^{2 r}\right)^{\frac{1}{2 r}} \\
& +\left(E \sup _{0 \leq t \leq T}\left|\int_{0}^{t} \mathbb{I}_{s \geq \tau} A^{1-\rho} e^{-A(t-s)}\left(g\left(\zeta^{1}(s)\right)-g\left(\zeta^{2}(s)\right)\right) \mathrm{d} s\right|^{2 r}\right)^{\frac{1}{2 r}} \\
& +\left(E \sup _{0 \leq t \leq T}\left|\int_{0}^{t} \mathbb{I}_{s \geq \tau} A^{1-\rho} e^{-A(t-s)}\left(G\left(\zeta^{1}(s)\right)-G\left(\zeta^{2}(s)\right)\right) \mathrm{d} w(s)\right|^{2 r}\right)^{\frac{1}{2 r}} \\
= & I+I I+I I I .
\end{aligned}
$$

Then from the assumptions

$$
I \leq c(M+1)^{2} \frac{T^{\rho}}{\rho}\left(E \sup _{0 \leq t \leq T}\left|A^{1-\rho}\left(\zeta^{1}(t)-\zeta^{2}(t)\right)\right|^{2 r}\right)^{\frac{1}{2 r}} .
$$


Similarly

$$
I I \leq c K \frac{T^{\rho-\rho^{\prime}}}{\rho-\rho^{\prime}}\left(E \sup _{0 \leq t \leq T}\left|A^{1-\rho}\left(\zeta^{1}(t)-\zeta^{2}(t)\right)\right|^{2 r}\right)^{\frac{1}{2 r}} .
$$

Then, using again Appendix 1, with

$$
\Phi(s)=\mathbb{I}_{s \geq \tau} A^{1-\rho}\left(G\left(\zeta^{1}(s)\right)-G\left(\zeta^{2}(s)\right)\right)
$$

and the same value of $\beta$ as above, we obtain

$$
I I I \leq C_{r, \rho, \varepsilon^{\prime}} K T^{\rho+\varepsilon^{\prime}-\frac{1}{2}}\left(E \sup _{0 \leq t \leq T}\left|A^{1-\rho}\left(\zeta^{1}(t)-\zeta^{2}(t)\right)\right|^{2 r}\right)^{\frac{1}{2 r}}
$$

So we have obtained

where

$$
\|\left|z_{1}-z_{2}\right|||_{T} \leq\left. C T^{\delta}||\left|\zeta_{1}-\zeta_{2}\right|\right|_{T}
$$

$$
\delta=\min \rho-\rho^{\prime}, \rho+\varepsilon^{\prime}-\frac{1}{2}
$$

and $C$ depends on all constants $\rho, \rho^{\prime}, \varepsilon^{\prime}, M, K, T_{0}$. Taking $T$ such that

$$
C T^{\delta}<1
$$

we obtain that $\mathcal{T}$ is a contraction in the space $\mathcal{H}_{T}$. Hence, we obtain a unique solution of $(8.9)$ in $\mathcal{H}_{T}$. We can extend such a solution a finite number of times to get a solution $y$ in $\mathcal{H}_{T_{0}}$, which is necessarily unique.

Applying the first part of the argument with $\zeta=y$, we obtain easily

$$
\|\| y \|_{T} \leq C\left[(1+M)^{2}+\left(E\left|A^{1-\rho} y_{\tau}\right|^{2 r}\right)^{\frac{1}{2 r}}\right]
$$

where the constant $C$ depends on $\rho, \rho^{\prime}, \varepsilon^{\prime}, K, T_{0}$. We can proceed in the same way, to evaluate a bound on intervals of time of length $T$, and derive the estimate (8.10). The last part of the statement follows immediately from the formula (8.9), applying Theorem 7.1, with the adequate value of $\beta$, and estimating conveniently the integral with $g$. The proof has been completed.

\section{REFERENCES}

[1] A. Bensoussan, Stochastic Navier Stokes Equations. Acta Appl. Math. 38 (1995) 267-304.

[2] A. Bensoussan and R. Temam, Equations stochastiques du type Navier-Stokes. J. Func. Anal. 13 (1973) 195-222.

[3] G. Da Prato, J. Zabczyk, Stochastic Equations in Infinite Dimensions, Cambridge University Press, Cambridge (1992).

[4] F. Flandoli and D. Gatarek, Martingale and Stationary Solutions for Navier-Stokes Equations, Preprints di Matematica - $\mathrm{n}^{\circ} 14$ (1994).

[5] N. Ikeda and S. Watanabe, Stochastic Differential Equations and Diffusion Processes, North Holland, Amsterdam (1981).

[6] I. Karatzas and S.E. Shereve, Brownian Motion and Stochastic Calculus, Springer-Verlag, New York (1988).

[7] J.L. Lions, Quelques méthodes de résolution des problèmes aux limites non linéaires, Dunod, Paris (1969).

[8] R. Temam, Navier-Stokes Equations, Theory and Numerical Analysis, North Holland (1977).

[9] W. von Wahl, The Equations of Navier-Stokes and Abstract Parabolic Equations, Aspects of Mathematics, Fr. Viewig \& Sohn, Braunschweig/Wiesbaden (1985). 